\title{
Discovery and Dynamical Analysis of an Extreme Trans-Neptunian Object with a High Orbital Inclination
}

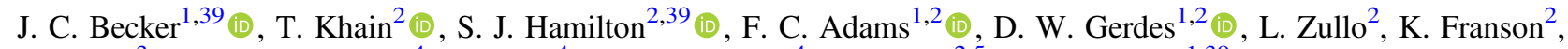
S. Millholland ${ }^{3}$ (1), G. M. Bernstein ${ }^{4}$, M. Sako ${ }^{4}$, P. Bernardinelli ${ }^{4}$, K. Napier ${ }^{2,5}$, L. Markwardt ${ }^{1,39}$, Hsing Wen Lin (林省文) $)^{2}$ (1), W. Wester ${ }^{6}$, F. B. Abdalla ${ }^{7,8}$, S. Allam ${ }^{6}$, J. Annis ${ }^{6}$ (D) S. Avila ${ }^{9}$, E. Bertin ${ }^{10,11}$, D. Brooks ${ }^{7}$, A. Carnero Rosell ${ }^{12,13}$, M. Carrasco Kind ${ }^{14,15}$, J. Carretero ${ }^{16}$, C. E. Cunha ${ }^{17}$, C. B. D’Andrea ${ }^{4}$, L. N. da Costa ${ }^{12,13}$, C. Davis ${ }^{17}$, J. De Vicente ${ }^{18}$, H. T. Diehl ${ }^{6}$, P. Doel ${ }^{7}$, T. F. Eifler ${ }^{19,20}$, B. Flaugher ${ }^{6}$, P. Fosalba ${ }^{21,22}$, J. Frieman ${ }^{6,23}$, J. García-Bellido ${ }^{24}$ (1) , E. Gaztanaga $^{21,22}$, D. Gruen ${ }^{17,25}$, R. A. Gruendl ${ }^{14,15}$ (D) , J. Gschwend ${ }^{12,13}$, G. Gutierrez ${ }^{6}$, W. G. Hartley ${ }^{7,26}$, D. L. Hollowood ${ }^{27}$, K. Honscheid ${ }^{28,29}$,

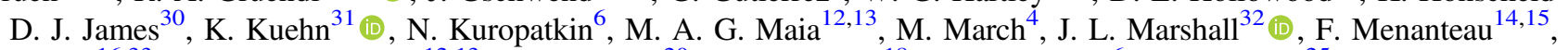
R. Miquel ${ }^{16,33}$ (1) R. L. C. Ogando ${ }^{12,13}$, A. A. Plazas ${ }^{20}$ (i) E. Sanchez $^{18}$ (i) , V. Scarpine ${ }^{6}$, R. Schindler ${ }^{25}$, I. Sevilla-Noarbe ${ }^{18}$,

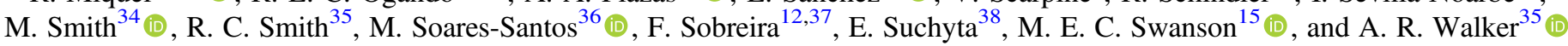
(DES Collaboration)

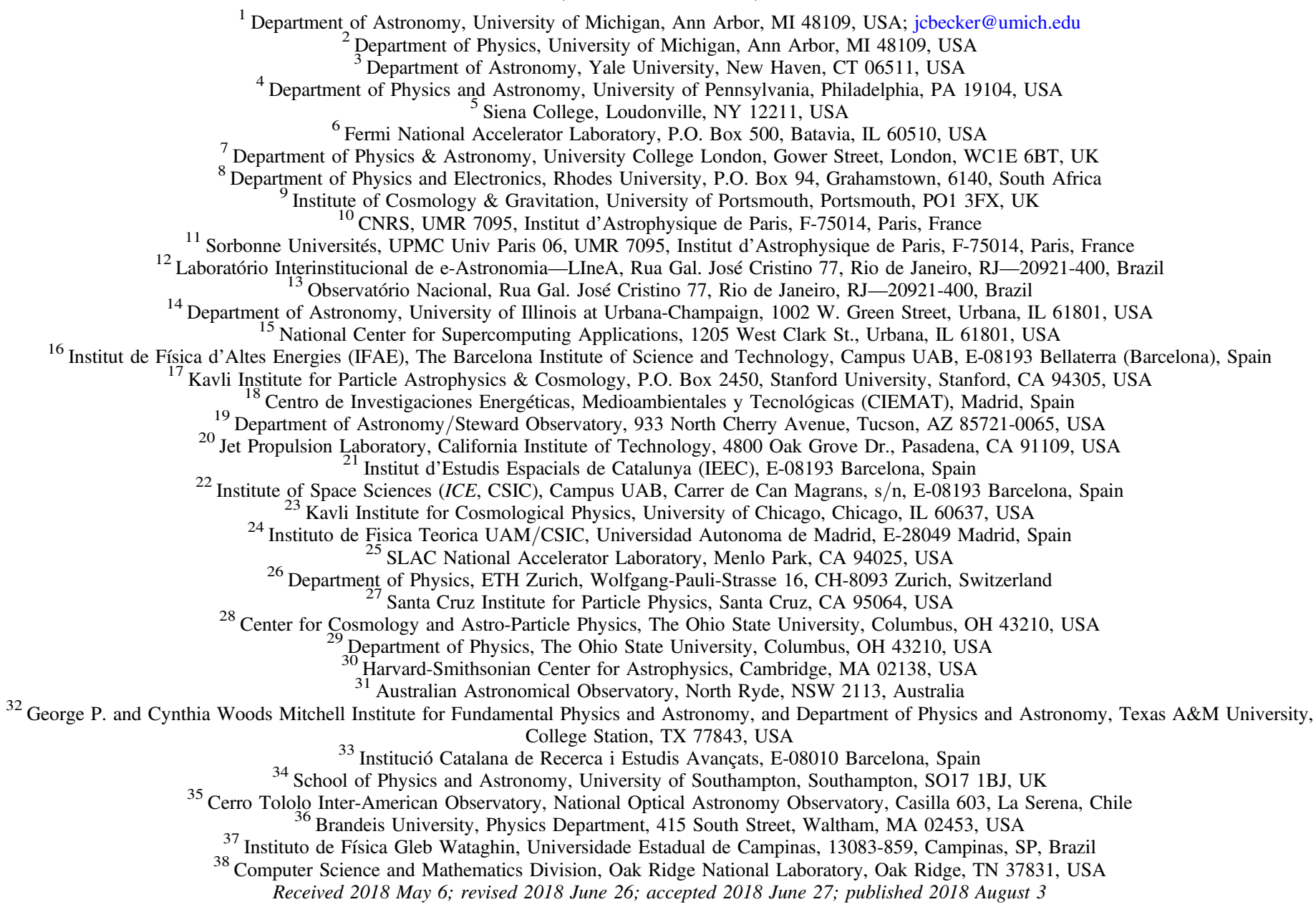

\begin{abstract}
We report the discovery and dynamical analysis of $2015 \mathrm{BP}_{519}$, an extreme trans-Neptunian object (TNO) detected by the Dark Energy Survey at a heliocentric distance of $55 \mathrm{au}$, perihelion of $\sim 36 \mathrm{au}$, and absolute magnitude $H_{r}=4.3$. The current orbit, determined from a 1110 day observational arc, has a semimajor axis $a \approx 450$ au, eccentricity $e \approx 0.92$, and inclination $i \approx 54^{\circ}$. With these orbital elements, $2015 \mathrm{BP}_{519}$ is the most extreme TNO discovered to date, as quantified by the reduced Kozai action, $\eta_{0}=\left(1-e^{2}\right)^{1 / 2} \cos i$, which is a conserved quantity at fixed semimajor axis $a$ for axisymmetric perturbations. We discuss the orbital stability and evolution of this object and find that, under the influence of the four known giant planets, $2015 \mathrm{BP}_{519}$ displays rich dynamical behavior, including rapid diffusion in semimajor axis and more constrained variations in eccentricity and
\end{abstract}

\footnotetext{
${ }^{39}$ NSF Graduate Research Fellow.
} 
inclination. We also consider the long-term orbital stability and evolutionary behavior within the context of the Planet Nine hypothesis and find that $2015 \mathrm{BP}_{519}$ adds to the circumstantial evidence for the existence of this proposed new member of the solar system, as it would represent the first member of the population of high- $i$, $\varpi$-shepherded TNOs.

Key words: Kuiper Belt objects: individual (2015 BP519) - planets and satellites: dynamical evolution and stability

Supporting material: interactive figures

\section{Introduction}

The most extreme members of any dynamical class of solar system objects serve as particularly acute test cases for theories of our solar system's formation and evolution. In particular, trans-Neptunian objects (TNOs) with very large semimajor axes probe the most distant observable regions of the solar system, aiding in revealing the migration histories of the giant planets. Very high inclination TNOs and centaurs mostly remain puzzling. Both classes of objects may also be dynamically influenced by distant, yet-unseen perturbers. Indeed, the apparent clustering in orbital and physical space of the so-called "extreme TNOs" (ETNOs) with $a>250 \mathrm{au}$ and perihelion distances $q>30$ au was used by Batygin \& Brown (2016a) to argue for the existence of a distant superEarth known as Planet Nine.

The 13 currently known ETNOs have an average orbital inclination of $17^{\circ} .3$. The most highly inclined of these objects, $2013 \mathrm{RF}_{98}$, was discovered in our earlier work (Dark Energy Survey Collaboration et al. 2016) and has an inclination of $29^{\circ} .6$, consistent with other members of the scattered disk population. In this work, we report the discovery by the Dark Energy Survey (DES) of $2015 \mathrm{BP}_{519}$, a TNO with a semimajor axis of 450 au (the sixth-largest among known TNOs), an eccentricity of 0.92 , and a remarkable inclination of $54^{\circ}$. The orbital elements of this object make it the "most extreme" of the ETNOs, in a sense that we make precise in Section 3.2. With a perihelion distance of $q=35.249 \pm 0.078$ au, it may also be the first purely trans-Neptunian member of the Planet Nine-induced high-inclination population first predicted in Batygin \& Morbidelli (2017).

Objects in the outer solar system populate several distinct dynamical categories (Gladman et al. 2008). Cold classical Kuiper Belt objects (CKBOs) are dynamically cool, with perihelion distances greater than $40 \mathrm{au}$, low orbital eccentricities, and low orbital inclinations (Tegler \& Romanishin 2000; Elliot et al. 2005). The orbits of these objects are not controlled by dynamical interactions with Neptune, and they may originate from material left over from the formation of the solar system. On the contrary, hot classical KBOs, as well as resonant KBOs, are believed to have been placed in the transNeptunian region from smaller original heliocentric distances. Another class of objects has orbits that are perturbed significantly through scattering interactions with Neptune (Duncan \& Levison 1997; Gladman et al. 2002). Yet another set of objects has high eccentricities but also sufficiently large perihelia that it is not influenced by either scattering or resonant interactions with Neptune.

Recently, a new subset of objects has attracted considerable attention. The TNOs with semimajor axes $a>150 \mathrm{au}$ and perihelia distances beyond $30 \mathrm{au}$ were found in Trujillo \& Sheppard (2014) to exhibit a clustering in their argument of perihelion, $\omega$. Batygin \& Brown (2016a) subsequently noted that this clustering persists in physical space (as measured by the longitude of perihelion $\varpi$, where $\varpi=\omega+\Omega$, where $\Omega$ is the longitude of the ascending node). Trujillo \& Sheppard (2014) noted that one explanation for the clustering might be a ninth planet, and Batygin \& Brown (2016a) suggested that the existence of a ninth planet of about 10 Earth masses in the outer solar system could explain the apparent alignment of large semimajor axis objects (Batygin \& Brown 2016a). The motions of objects with $a>250$ au would in this case be dominated by Planet Nine, while TNOs falling in the intermediate regime, with $a=150-250 \mathrm{au}$, might experience differing degrees of influence from Planet Nine. The TNOs with $a>250$ au constitute the ETNOs. The evidence and consequences of the Planet Nine hypothesis have been explored in the literature from both dynamical and observational perspectives (Batygin \& Brown 2016b; Bromley \& Kenyon 2016; Chen et al. 2016; de la Fuente Marcos \& de la Fuente Marcos 2016; Holman \& Payne 2016a, 2016b; Kenyon \& Bromley 2016; Li \& Adams 2016; Malhotra et al. 2016; Mustill et al. 2016; Sheppard \& Trujillo 2016; Batygin \& Morbidelli 2017; Becker et al. 2017; Hadden et al. 2018; Millholland \& Laughlin 2017; Parker et al. 2017; Saillenfest et al. 2017; Shankman et al. 2017b; Eriksson et al. 2018; Khain et al. 2018). The Planet Nine hypothesis has been invoked to explain the detachment of perihelia distance for the most distant class of TNOs (Khain et al. 2018), the $6^{\circ}$ solar obliquity (Bailey et al. 2016; Gomes et al. 2017), and the existence of highly inclined objects in the outer solar system (Batygin \& Brown 2016b). The subset of objects discovered so far to have semimajor axes greater than 250 au and perihelion distances greater than 30 au (the ETNOs) includes 2003 VB $_{12}$ (known as Sedna; Brown et al. 2004), 2004 VN $_{112}$ (MPC), 2007 $\mathrm{TG}_{422}$ (MPC), $2010 \mathrm{~GB}_{174}$ (Chen et al. 2013), $2012 \mathrm{VP}_{113}$ (Trujillo \& Sheppard 2014), $2013 \mathrm{FT}_{28}$ (Sheppard \& Trujillo 2016), $2013 \mathrm{RF}_{98}$ (MPC), $2013 \mathrm{SY}_{99}$ (Bannister et al. 2017), $2014 \mathrm{FE}_{72}$ (Sheppard \& Trujillo 2016), 2014 SR $_{349}$ (Sheppard \& Trujillo 2016), $2015 \mathrm{GT}_{50}$ (Shankman et al. 2017a), $2015 \mathrm{KG}_{163}$ (Shankman et al. 2017a), and $2015 \mathrm{RX}_{245}$ (Shankman et al. 2017a). The orbital elements of these objects are listed in the Appendix for reference. These objects have inclinations ranging from nearly zero up to a maximum of about $30^{\circ}$.

The orbital inclinations of these high- $a$ objects are of particular interest dynamically. Gladman et al. (2009) discovered $2008 \mathrm{KV}_{42}$ (Drac), the first retrograde Centaur (where a Centaur is an object with a semimajor axis between 5 and $30 \mathrm{au}$, placing its orbit in the region of the solar system containing the gas giants). This object does not appear to be primordial and could imply the existence of a reservoir of highinclination TNOs. The discovery of the retrograde centaur $2011 \mathrm{KT}_{19}$ (Niku; Chen et al. 2016) added to the small collection of such objects and suggested that they may cluster in a common orbital plane.

Batygin \& Brown (2016a) predicted that Planet Nine could create such a supply of objects by sourcing them from a more distant population of high-inclination orbits, which are in turn generated by Planet Nine. Batygin \& Morbidelli (2017) 
presented a dynamical model for the orbital evolution of highinclination, long-period ( $a>250 \mathrm{au}$ ) objects and compared the model to the existing high- $a$, high- $i$ objects. However, the objects then known to reside in that population have perihelia $q<30$ au and thus experience orbit crossing with the giant planets, complicating their ability to test the Planet Nine hypothesis. To better test this particular prediction of the Planet Nine model, high- $a$, high- $i$ objects with perihelion $q>30$ au are needed.

Apart from the Planet Nine debate, the continued discovery of new objects in the outer solar system enables a better understanding of how the solar system arrived at its present state. For this reason, many groups have conducted surveys to increase the census of objects known in the outer solar system and better understand their properties (including Millis et al. 2002; Elliot et al. 2005; Lellouch et al. 2010; Müller et al. 2010; Lim et al. 2010; Fornasier et al. 2013; Bannister et al. 2016; Lin et al. 2016). Data from other surveys or archival data sets have also been utilized to enable solar system science (Fuentes \& Holman 2008; Solontoi et al. 2012; Ahn et al. 2014). The DES (Dark Energy Survey Collaboration et al. 2016) follows in these footsteps, enabling the study of new populations, including high-inclination objects like 2015 $\mathrm{BP}_{519}$.

This paper is organized as follows. We start with a description of the discovery of $2015 \mathrm{BP}_{519}$ by DES in Section 2. Given the extreme status of this object, Section 3 considers its dynamical status using a secular approach, starting with an analytic treatment of the problem. The dynamics of this object are rich and complicated, so that a complete characterization requires full $\mathrm{N}$-body numerical simulations to include interactions with Neptune and the other giant planets, as well as other complexities. These numerical simulations are presented in Section 4 for the dynamics of $2015 \mathrm{BP}_{519}$ in the context of the currently known solar system. Section 5 then considers the dynamics of this new object in the presence of the proposed Planet Nine. The paper concludes with a discussion of the implications (Section 6) and a summary of the results (Section 7).

\section{Discovery of $2015 \mathrm{BP}_{519}$}

The DES (Dark Energy Survey Collaboration et al. 2016) is an optical survey targeting nearly $5000{ }^{\circ 2}$ of sky. It uses the Dark Energy Camera (DECam; Flaugher et al. 2015) on the $4 \mathrm{~m}$ Blanco telescope at the Cerro Tololo Inter-American Observatory in Chile. DECam is a prime-focus imager on Blanco with a $3 .^{\circ}$ field of view and a focal plane consisting of $622 K \times 4 K$ red-sensitive science CCDs. The DES saw first light in 2012, and the nominal survey period of 520 nights over 5 yr ran from 2013 August through 2018 February. During this time, the DES operated in two survey modes. The Wide Survey observes the full survey area roughly twice per year in each of the grizY bands. The Supernova Survey (Bernstein et al. 2012) consists of $103 .^{\circ 2}$ regions that are observed roughly every 6 days in the griz bands. Due to the large survey area, high repetition, and deep limiting magnitude for single-epoch exposures $(r \sim 23.8 \mathrm{mag})$, the DES has many applications in addition to its main cosmological objectives (Dark Energy Survey Collaboration et al. 2016). It is well suited for solar system science, in particular the study of high-inclination populations.

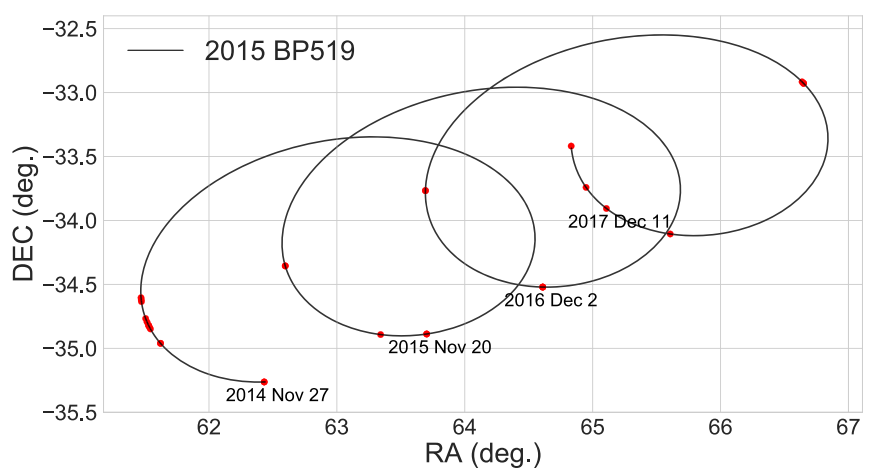

Figure 1. Trajectory of $2015 \mathrm{BP}_{519}$ over its measured four-opposition arc. Larger red dots along the trajectory indicate points at which it was observed by DES.

In this paper, we report the discovery of $2015 \mathrm{BP}_{519}$, which has the largest semimajor axis of any object yet found by the DES and the highest inclination of any known ETNO. It was first detected at a heliocentric distance of 55 au in the same set of observations from 2013 to 2016 that were used to discover the dwarf planet candidate $2014 \mathrm{UZ}_{224}$ (Gerdes et al. 2017). The original detection of $2015 \mathrm{BP}_{519}$ came from a differenceimaging analysis of the wide-field images (using software from Kessler et al. 2015). Transient objects are found by image subtraction. Artifacts and low-quality detections are rejected using the techniques described in Goldstein et al. (2015). The surviving sources are compiled into a catalog of measurements, each of which corresponds to a transient at one epoch. From those, TNOs are extracted by identifying pairs of detections within 60 nights of each other whose angular separation is consistent with what would be expected for an object with perihelion $q>30$ au given Earth's motion. These pairs are linked into chains of observations that correspond to the same object by testing the goodness of fit of the best-fit orbit for each chain. A reduced $\chi^{2} / N<2$ (Bernstein \& Khushalani 2000) is considered a detection of a TNO.

Although $2015 \mathrm{BP}_{519}$ was originally identified using data from observing campaigns 2-4, we have obtained additional observations in two ways. First, some of the subsequent planned DES exposures provided additional serendipitous observations of this object. Second, we performed three targeted observations on 2017 February 2 and 2017 August 6-7. The result is a series of 30 observations over four oppositions between 2014 November 27 and 2018 February 15, shown in Figure 1. We computed astrometric positions using the WCSFIT software described in Bernstein et al. (2017), which provides astrometric solutions referenced to the Gaia DR1 catalog (Gaia Collaboration et al. 2016). This includes corrections for the effects of tree-ring and edge distortions on the DECam CCDs, as well as for chromatic terms from lateral color and differential atmospheric refraction. We obtain barycentric osculating orbital elements using the method of Bernstein \& Khushalani (2000). For consistency with the orbital elements and uncertainties used in the simulation results presented below, our fit uses the 27 observations available through 2017 December 11. The resulting fit has a $\chi^{2}$ of 48.2 for 48 degrees of freedom and a mean residual of 29 mas. These orbital elements are shown in Table 1. The inclination and orbital orientation of $2015 \mathrm{BP}_{519}$ relative to those of the other ETNOs are also visualized in Figure 2 (where the orbital 
Table 1

Orbital Elements of $2015 \mathrm{BP}_{519}$

\begin{tabular}{ll}
\hline \hline Parameter & Value \\
\hline$a$ & $448.99 \pm 0.49 \mathrm{au}$ \\
$e$ & $0.92149 \pm 0.00009$ \\
$i$ & $54.1107 \pm 0.00001$ \\
$\omega$ & $348.058 \pm 0.00136$ \\
$\Omega$ & $135.2131 \pm 0.00010$ \\
Time of perihelion (JD) & $2,473,015.55 \pm 0.56$ \\
Perihelion & $35.249 \pm 0.078 \mathrm{au}$ \\
Aphelion & $862.733 \pm 0.972 \mathrm{au}$ \\
Orbital period & $9513.84 \pm 15.42 \mathrm{yr}$ \\
Absolute magnitude & $H_{r}=4.3$ \\
$g-r$ (mag) & $0.79 \pm 0.17$ \\
$r-i$ (mag) & $0.19 \pm 0.12$ \\
$r-z$ (mag) & $0.42 \pm 0.15$ \\
$i-z$ (mag) & $0.23 \pm 0.15$ \\
\hline
\end{tabular}

Note. The $2015 \mathrm{BP}_{519}$ barycentric osculating elements at epoch 2,456,988.83, based on 27 observations over a 1110 day arc from 2014 November 27 to 2017 December 12 . Object $2015 \mathrm{BP}_{519}$ has a mean anomaly 358.34 and will reach perihelion on 2058 October 14 .

elements used for the other plotted ETNOs are given in the Appendix, Table 3).

These 27 observations of $2015 \mathrm{BP}_{519}$ include eight measurements in the $g$ band, nine in the $r$ band, six in the $i$ band, and four in the $z$ band. Few of these observations were taken in close temporal proximity. To compute the colors of this object, we therefore compute the corresponding absolute magnitude $H$ of each measurement to correct for the varying object-Sun and object-Earth distances, as well as differences in observational phase. The $g-r$ color, for example, is then computed as $\left\langle H_{g}\right\rangle-\left\langle H_{r}\right\rangle$, and its uncertainty is $\left(\left\langle H_{g}^{2}\right\rangle+\left\langle H_{r}^{2}\right\rangle\right)^{1 / 2}$. The moderately red $g-r$ and $r-z$ colors are consistent with the values measured in Pike et al. (2017) for objects identified as dynamically excited.

For TNOs with magnitudes in the range $H \sim 2-4$, measured visual albedos have been found to range between 0.07 and 0.21 (Brucker et al. 2009; Lellouch et al. 2013; Fraser et al. 2014; Gerdes et al. 2016; Holman et al. 2018). With $H_{r}=4.3$, the diameter of $2015 \mathrm{BP}_{519}$ could range from 400 to $700 \mathrm{~km}$, depending on whether the albedo falls near the high or low end of this range.

Because the DES area lies predominantly out of the ecliptic, the status of $2015 \mathrm{BP}_{519}$ as the highest-inclination TNO of those with semimajor axis $a>250$ au and perihelion $q>30$ au must be considered in the context of possible bias of the DES selection function. To explore this issue, we simulate an ensemble of clones of $2015 \mathrm{BP}_{519}$ and test their recoverability in the DES TNO search pipeline. The orbital elements of these clones are drawn from the observed posteriors provided in Table 1 but with the inclination angle $i$ drawn from a uniform distribution between $0^{\circ}$ and $180^{\circ}$. We then compute the orbits of these objects and where the clones would fall on the nights DES observed.

Using these synthetic orbital elements, we first remove any object that is not detectable by DES because it is either too faint or outside the survey area. We then compute the position of each remaining clone at the time of every DES exposure belonging to the data set in which $2015 \mathrm{BP}_{519}$ was discovered and determine which clones could be linked together into an orbit. The clones that could be identified as candidates are those

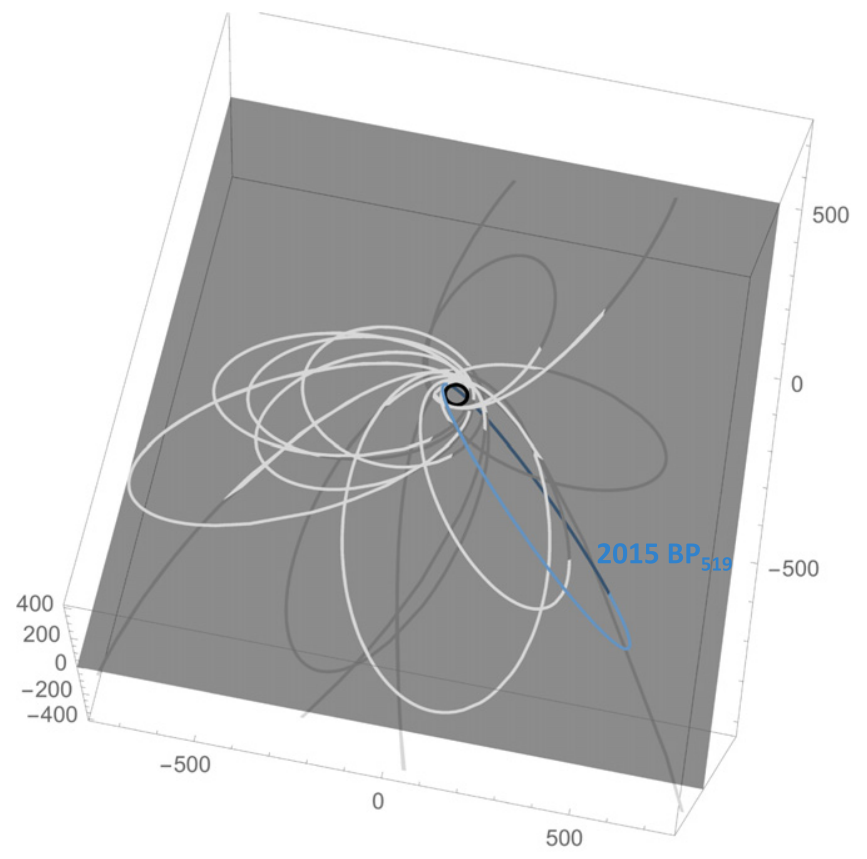

Figure 2. Visual representation of the orbit of $2015 \mathrm{BP}_{519}$, plotted with the other ETNOs as comparisons. For each orbit, the darker regions on the curve denote where an object falls below the plane of the solar system. Of any ETNO discovered to date, $2015 \mathrm{BP}_{519}$ has the highest inclination. The full interactive 3D orbit visualization can also be found at https://smillholland.github.io/ BP519/.

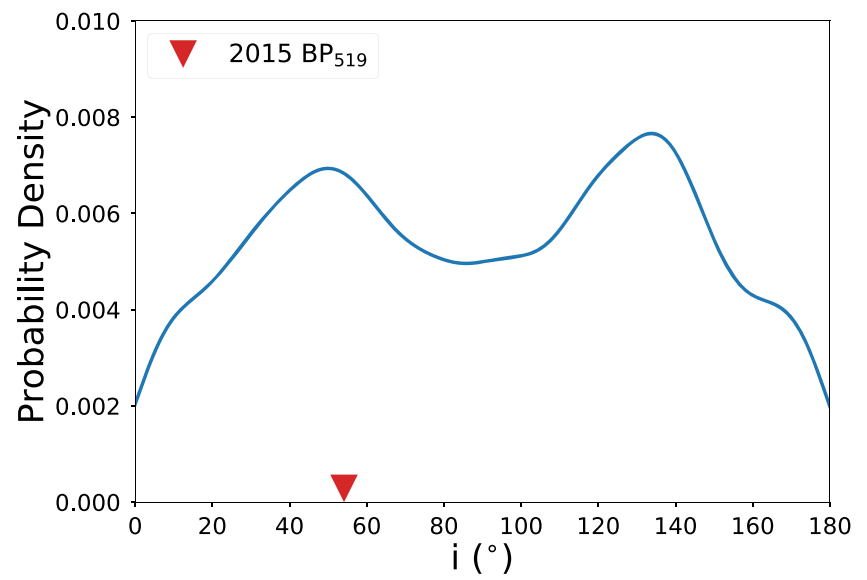

Figure 3. The DES selection function for the discovery of objects with the orbital elements of $2015 \mathrm{BP}_{519}$ but varying inclination angles. The value for $2015 \mathrm{BP}_{519}$ is shown as the red triangle. The probability distribution is normalized so that the area under the curve is unity.

with at least three observations on three different nights separated by less than 60 nights and observations on at least five different nights in total.

The distribution of clones that survive this process, and hence are potentially detectable, is presented in Figure 3. This plot thus shows the sensitivity function for objects with the orbital parameters $(a, e, \omega, \Omega)$ of $2015 \mathrm{BP}_{519}$ but with varying orbital inclinations and mean anomalies. The resulting sensitivity function shows some structure but is not heavily biased toward the observed inclination angle of $2015 \mathrm{BP}_{519}$.

Object $2015 \mathrm{BP}_{519}$ has the highest inclination of any known TNO (defined as objects with $q>30 \mathrm{au}$ ). It also has an extreme eccentricity (0.92). Figure 4 compares the inclination and eccentricity occurrences by semimajor axis of the regular 

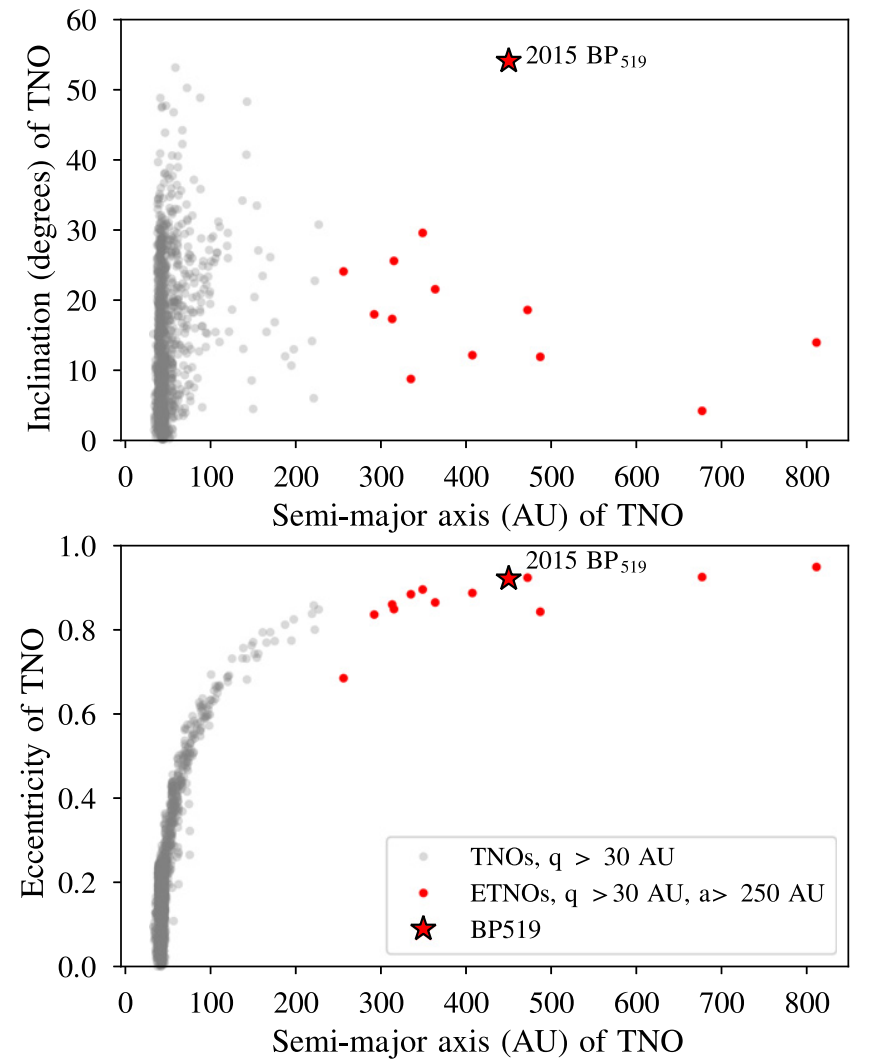

Figure 4. Distributions of inclinations (top panel) and eccentricities (bottom panel) for the two populations of TNOs considered in this work: all objects with perihelia distances greater than $30 \mathrm{au}$ and the subset of those with semimajor axes greater than $250 \mathrm{au}$. Orbits of known objects are fit from observations posted to the Minor Planet Center database. Object 2015 BP $_{519}$ represents the tail of the inclination distribution of the known TNOs, as well as the upper limit of eccentricities populated by TNOs.

and extreme ( $a>250 \mathrm{au}$ ) TNO populations. Compared to the other known TNOs, $2015 \mathrm{BP}_{519}$ has the largest orbital inclination. Since the number of known ETNOs is small, however, is it unclear where $2015 \mathrm{BP}_{519}$ 's inclination places it in the true distribution of ETNO inclinations. For regular TNOs (objects with perihelion distance $q>30$ au but any semimajor axis), for which nearly 1500 have been discovered, $2015 \mathrm{BP}_{519}$ is the most extreme and seems to lie at the upper tail of the inclination distribution of known objects; among TNOs, 2015 $\mathrm{BP}_{519}$ has the highest currently measured value, but this population is by no means complete.

In Figure 5, we plot a sensitivity histogram computed with the method described above but for objects with the orbital parameters $(a, e, i)$ of $2015 \mathrm{BP}_{519}$ and varying $\omega$ and $\Omega$. As was true for the previous sensitivity function, the final sensitivity histogram shows some structure in each orbital angle of interest but is not heavily biased toward the measured angles of 2015 $\mathrm{BP}_{519}$ (which happen to be consistent with the region of clustering that was used to predict Planet Nine, as will be discussed further in later sections of this paper; Trujillo \& Sheppard 2014; Batygin \& Brown 2016a).

\section{Characterization of $2015 \mathrm{BP}_{519}$}

As a starting point, we consider the dynamical behavior of $2015 \mathrm{BP}_{519}$ using a secular treatment. The basic approach is outlined and compared with numerical $N$-body experiments in
Section 3.1, and this formalism is used to elucidate the extreme nature of this object in Section 3.2.

\subsection{Secular Dynamics}

A secular approach averages over the mean motion of solar system objects and thus allows for a simplified treatment of the long-term dynamics. Kozai (1962) provided secular equations for the orbital evolution of small bodies with high inclinations and eccentricities in the presence of an inner perturber. Here we want to describe the behavior of $2015 \mathrm{BP}_{519}$, which orbits outside a system of four interior perturbers (namely, the known giant planets). The contribution from the terrestrial planets is negligible in this context. We can write the mean perturbing function $R_{m}$ for a test particle evolving in the presence of a set of inner planets in the form

$$
\begin{aligned}
R_{m}= & \frac{G}{16 a^{3}\left(1-e^{2}\right)^{3 / 2}}\left[\sum_{j}^{N}\left(m_{j} a_{j}^{2}\right)(1+3 \cos 2 i)\right. \\
& +\frac{9 \sum_{j}^{N}\left(m_{j} a_{j}^{4}\right)\left(2+3 e^{2}\right)(9+20 \cos 2 i+35 \cos 4 i)}{512 a^{2}\left(1-e^{2}\right)^{2}} \\
& \left.+\frac{9 \sum_{j}^{N}\left(m_{j} a_{j}^{4}\right) 40 e^{2}(5+7 \cos 2 i) \cos 2 \omega \sin ^{2} i}{512 a^{2}\left(1-e^{2}\right)^{2}}\right],
\end{aligned}
$$

where the effects of the inner planets are included here as a mean moment of inertia (Gallardo et al. 2012). In this expression, $G$ is the gravitational constant $\left(G=4 \pi^{2}\right.$; we work in units of solar mass, au, and $\mathrm{yr}),(a, e, i)$ are the orbital elements of the test particle, $\sum_{j}^{N}\left(m_{j} a_{j}^{2}\right)$ is the moment of inertia in the direction out of the plane containing the giant planets, and the label $j$ denotes each giant planet under consideration.

From this secular Hamiltonian, we can derive an expression for the time evolution of the inclination angle using Lagrange's planetary equations, which takes the form

$$
\begin{aligned}
\frac{d i}{d t}= & -\tan (i / 2)\left(n a^{2} \sqrt{1-e^{2}}\right)^{-1}\left(\frac{d R_{m}}{d \epsilon}+\frac{d R_{m}}{d \varpi}\right) \\
& -\left(n a^{2} \sqrt{1-e^{2}} \sin i\right)^{-1} \frac{d R_{m}}{d \Omega},
\end{aligned}
$$

where $n=\left(G M / a^{3}\right)^{1 / 2}$ is the mean motion, $M$ is the mass of the central body, and $\epsilon$ is the mean longitude at epoch. Combining the previous two expressions yields the equation of motion for $i$ :

$$
\frac{d i}{d t}=\frac{45 e^{2} G^{1 / 2} \sum_{j}^{N}\left(m_{j} a_{j}^{4}\right)}{1024 M^{1 / 2} a^{11 / 2}\left(1-e^{2}\right)^{4}}(5+7 \cos 2 i) \sin 2 i \sin 2 \omega .
$$

Analogous equations can also be constructed using the other Lagrange planetary equations, resulting in equations of motion for $d e / d t, d \omega / d t, d \Omega / d t$ (see Equations 7-11 of Gallardo et al. 2012), and $d a / d t=0$. Using the known (estimated) orbital elements for $2015 \mathrm{BP}_{519}$ (see Table 1) as initial conditions, we simultaneously solved these five equations of motion, resulting in a predicted secular evolution for 2015 

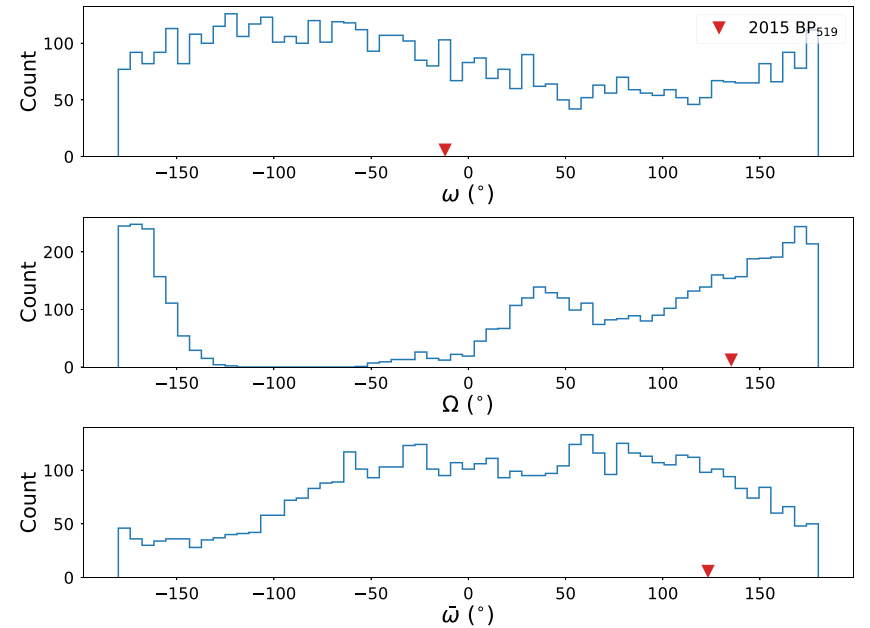

Figure 5. The DES selection function for the discovery of objects with the orbital elements $(a, e, i)$ of $2015 \mathrm{BP}_{519}$ but with varying angles $\omega, \Omega$. The observed values for $2015 \mathrm{BP}_{519}$ are shown as red triangles in each panel. For objects with the orbital elements $(a, e, i)$ of $2015 \mathrm{BP}_{519}$, the DES observation bias allows discovery of $\omega$ and $\Omega$ subtending most of the allowable ranges.

$\mathrm{BP}_{519}$ 's orbital evolution. This result is shown in Figure 6 as the solid red curve. The figure shows additional curves in gray corresponding to the orbital evolution computed for the same initial conditions but using full $\mathrm{N}$-body integrations instead of secular theory. These simulations are described in full in the following section, and their parameters are also summarized as Set 1 in Table 2. As a quick summary, these integrations are computed using the Mercury 6 integration package (Chambers 1999), using the hybrid symplectic and BulirschStoer (B-S) integrator and a timestep of 20 days. In these simulations, all four of the known giant planets are treated as active bodies (rather than being modeled using the $J_{2}$ approximation that is often used).

Figure 6 shows that the secular approximation provides a good order-of-magnitude description of the time evolution of the inclination angle, even though the secular approximation does not include the scattering interactions that lead to a slight divergence in the $N$-body simulations. Both the secular and $\mathrm{N}$-body treatments predict that, in the known solar system, the inclination of $2015 \mathrm{BP}_{519}$ will remain fairly well constrained around its presently observed value.

\subsection{The Extreme Nature of $2015 \mathrm{BP}_{519}$}

Although the orbit of $2015 \mathrm{BP}_{519}$ is highly unusual among known TNOs, we need a quantitative assessment of its properties relative to other TNOs of its dynamical class. Toward that end, we consider the Kozai Hamiltonian written in Delaunay coordinates (Thomas \& Morbidelli 1996), for which the action $H$ is defined as

$$
H=\sqrt{a\left(1-e^{2}\right)} \cos i .
$$

Note that this action is equivalent to the standard " $\mathcal{H}$ " variable in Delaunay coordinates (Murray \& Dermott 1999) and is a constant of the motion in the quadrupolar approximation. The action $L=\sqrt{a}$ will also be constant, as the Kozai Hamiltonian is averaged over the mean anomaly, is thus rotationally invariant, and thus depends only on action $G=\sqrt{a\left(1-e^{2}\right)}$ and coordinate $g=\omega$, with actions $L$ and $H$

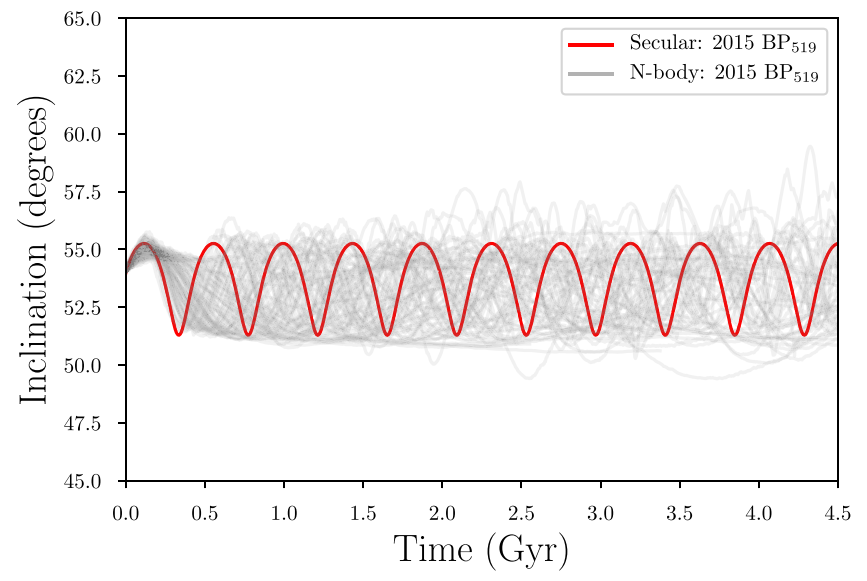

Figure 6. Future evolution of $2015 \mathrm{BP}_{519}$ (using its current-day best-fit orbital parameters as initial conditions) in the presence of the known solar system. The secular curve plotted as a solid red line was solved from the disturbing function (Equation (1)) and the best-fit orbital elements of $2015 \mathrm{BP}_{519}$. The numerical results, plotted as gray lines, are drawn from simulation Set 1, where the orbit of $2015 \mathrm{BP}_{519}$ is evolved in the presence of the known solar system for 4.5 Gyr. See Table 2 for more details on the simulation parameters.

being conserved. Next, we define a reduced Kozai action $\eta_{0}$, which has the form

$$
\eta_{0}=\sqrt{\left(1-e^{2}\right)} \cos i .
$$

As action $L$ is conserved for the Kozai Hamiltonian, this reduced form of action $H$ should also be conserved. Note that $\eta_{0}$ is the specific angular momentum vector in the direction out of the plane of the solar system (Kinoshita \& Nakai 1999; we follow the notation in Saillenfest et al. 2016). For sufficiently distant TNOs, the potential of the solar system is effectively axially symmetric (but not spherically symmetric), so that the $z$-component of angular momentum (but not total angular momentum) is conserved. In the known solar system, TNOs with constant semimajor axes are thus expected to evolve in $(i, e)$ space along contours of constant $\eta_{0}$. In Figure 7 , we overlay curves of constant $\eta_{0}$ on a plot comparing the $i, e$ of all TNOs and ETNOs discovered so far. Compared to previously discovered objects, $2015 \mathrm{BP}_{519}$ has the lowest $\eta_{0}$ value, which signifies its relative extremeness.

\section{Full Dynamics of $2015 \mathrm{BP}_{519}$ in the Known Solar System}

The analytic formulation presented in Section 3 classifies $2015 \mathrm{BP}_{519}$ as the most extreme of the ETNOs discovered in the outer solar system to date due to its high inclination, high eccentricity, and large semimajor axis. However, the secular approximation used in the previous section neglects the importance of interactions with Neptune, which will occur when $2015 \mathrm{BP}_{519}$ reaches its perihelion. The relatively small perihelion distance $(\approx 35 \mathrm{au})$ of $2015 \mathrm{BP}_{519}$ suggests that it will be subject to repeated strong interactions with Neptune, which will change the energy of its orbit by a factor of roughly $6 \times 10^{-6}$ per perihelion crossing (when this process can be modeled as a random walk; see Figure 1 of Duncan et al. 1987). The change in orbital energy will also lead to a change in the semimajor axis of the orbit, and as a result, the level curves presented in Figure 7 may not truly represent the evolution of $2015 \mathrm{BP}_{519}$ over extended spans of time. Instead, quantities that appear as constants of motion in the previous section $\left(\eta_{0}\right)$ will no longer be conserved, as $2015 \mathrm{BP}_{519}$ changes its orbital elements, in particular its semimajor axis, 
Table 2

Simulation Sets Used in This Work

\begin{tabular}{|c|c|c|c|c|c|c|c|}
\hline Set & Initial Timestep & Active Planets & $J_{2}$ & Abs. Radius & Backward Clones & Forward Clones & Details \\
\hline Set 1 & 20 days & 4 (JSUN) & $2 \times 10^{-7}$ & $4.65 \times 10^{-3} \mathrm{au}$ & 130 & 130 & No P9 \\
\hline Set 2 & 3000 days & $1(\mathrm{P9})$ & 0.00015244 & $20 \mathrm{au}$ & 0 & 1000 & Low $i^{\mathrm{a}}, \mathrm{P} 9^{\mathrm{b}}$ \\
\hline Set 3 & 3000 days & 1 (P9) & 0.00015244 & $20 \mathrm{au}$ & 0 & 1000 & $P 9^{\mathrm{b}}$ \\
\hline Set 5 & 20 days & 5 (JSUN, P9) & $2 \times 10^{-7}$ & $4.65 \times 10^{-3} \mathrm{au}$ & 0 & 130 & $\mathrm{P} 9^{\mathrm{b}}$ \\
\hline
\end{tabular}

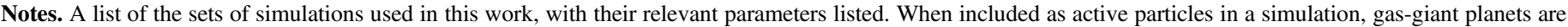

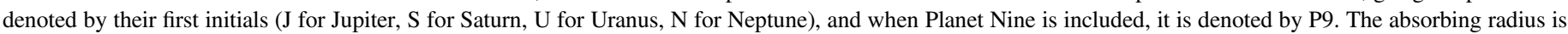

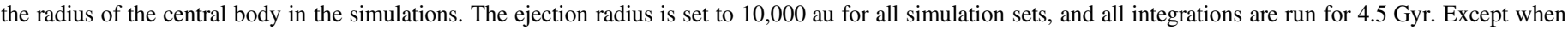

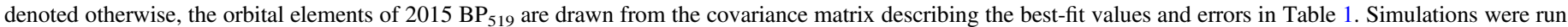

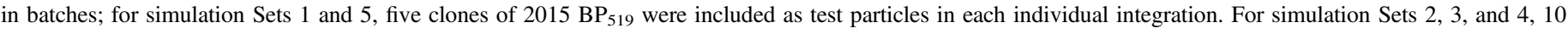
clones were included in each integration.

${ }^{a}$ Inclination of $2015 \mathrm{BP}_{519}$ was drawn from a half-normal distribution around $0^{\circ}$ with a width of $5^{\circ}$.

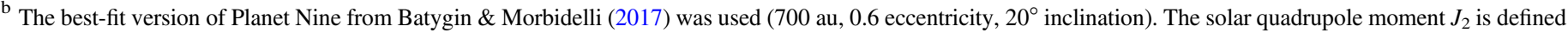
by Equation (6) when any giant planets are absorbed and set to the solar value otherwise (Pireaux \& Rozelot 2003).

due to interactions with Neptune. The true orbital evolution, being the result of a chaotic process, will also vary widely between trials in numerical integrations. As shown in Figure 6, the numerically computed orbital evolution does not perfectly match the secular expectation, and multiple integrations of the same object will give slightly different periods and amplitudes of evolution.

To fully test the effect of additional dynamics not encapsulated by the secular analysis of the previous section, we perform a suite of numerical $N$-body simulations using computing resources provided by Open Science Grid (Pordes et al. 2007; Sfiligoi 2008; Sfiligoi et al. 2009) through the Extreme Science and Engineering Discovery Environment (XSEDE) portal (Towns et al. 2014). These simulations include the new body $2015 \mathrm{BP}_{519}$ and all of the relevant known solar system objects (the case of Planet Nine is considered in the following section but is excluded from this initial set of simulations).

\subsection{Numerical Evolution of $2015 \mathrm{BP}_{519}$ in the Known Solar System}

The precession timescales and orbital evolution of 2015 $\mathrm{BP}_{519}$ can be tested more directly with numerical $N$-body simulations. To examine the complete evolution of $2015 \mathrm{BP}_{519}$ in the known solar system, we perform a suite of numerical integrations using the Mercury6 integration package (Chambers 1999). We exclude the terrestrial planets from the simulations but include the gas giants (Jupiter, Saturn, Uranus, and Neptune) as active, massive particles with their currently measured masses and orbital elements. We start with a timestep of 20 days, which is roughly $0.5 \%$ of Jupiter's orbital period. We use the hybrid symplectic and B-S integrator built into Mercury 6 and conserve energy to better than one part in $10^{9}$ over the course of the $4.5 \mathrm{Gyr}$ integrations. The orbital elements for $2015 \mathrm{BP}_{519}$ are drawn from the covariance matrix derived from the fit to the DES data. Fifty-two simulations are run of the solar system, each with five clones of $2015 \mathrm{BP}_{519}$. Half of these simulations are integrated forward in time from the current day, and the other half evolve back in time for 4.5 Gyr. Other parameters used for this set of simulations (which we call Set 1 in this work) are given in Table 2.

The results of these integrations are presented in Figure 8 and demonstrate that the semimajor axis of $2015 \mathrm{BP}_{519}$ diffuses

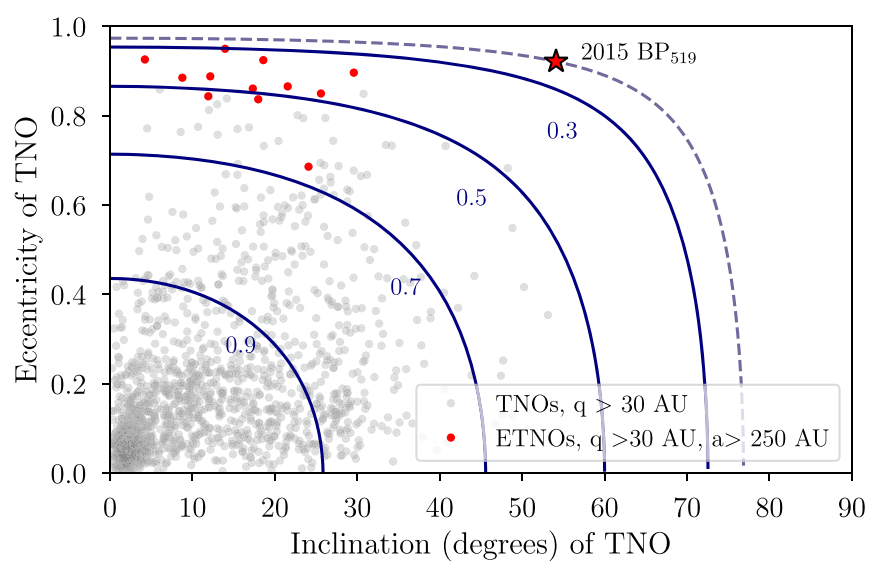

Figure 7. We plot curves of constant $\eta_{0}$ in (i,e) space (see Equation (5)). Also plotted (gray) are the orbital elements of all objects with perihelion distances outside of Neptune and data quality flags of 6 or better, as reported to the Minor Planet Center database (files downloaded 10/25/2017), and (red) the subset of those objects that also have a semimajor axis measured to be $a>250 \mathrm{au}$. With $\eta_{0}=0.2274,2015 \mathrm{BP}_{519}$ has the lowest value of $\eta_{0}$ out of any TNO with $q>30$ au that has been discovered thus far. This metric, which measures the extremeness of the $(i, e)$ of each object, characterizes $2015 \mathrm{BP}_{519}$ as the most extreme of the ETNOs.

widely in the presence of the known giant planets. The perihelion distance tends to remain fairly well confined near the initial value of even as the orbital energy changes due to repeated kicks from Neptune.

The Hamiltonian used in Section 3.2 requires that the semimajor axis of the particle remain roughly constant. From these simulations, it is clear that the semimajor axis of 2015 $\mathrm{BP}_{519}$ tends to change significantly over relatively rapid $\left(\sim 10^{6}-10^{7} \mathrm{yr}\right)$ timescales. As such, Equation (5) is a good model for $2015 \mathrm{BP}_{519}$ 's short-term dynamical behavior but not its long-term orbital evolution.

\subsection{Generating Highly Inclined Objects in the Known Solar System}

In the previous section, we used numerical simulations to determine the expected evolution of $2015 \mathrm{BP}_{519}$ in the presence of the four known gas giants. The results show that the inclination of $2015 \mathrm{BP}_{519}$ tends to be confined to within a range of roughly $5^{\circ}$. Although the semimajor axis diffuses over a 


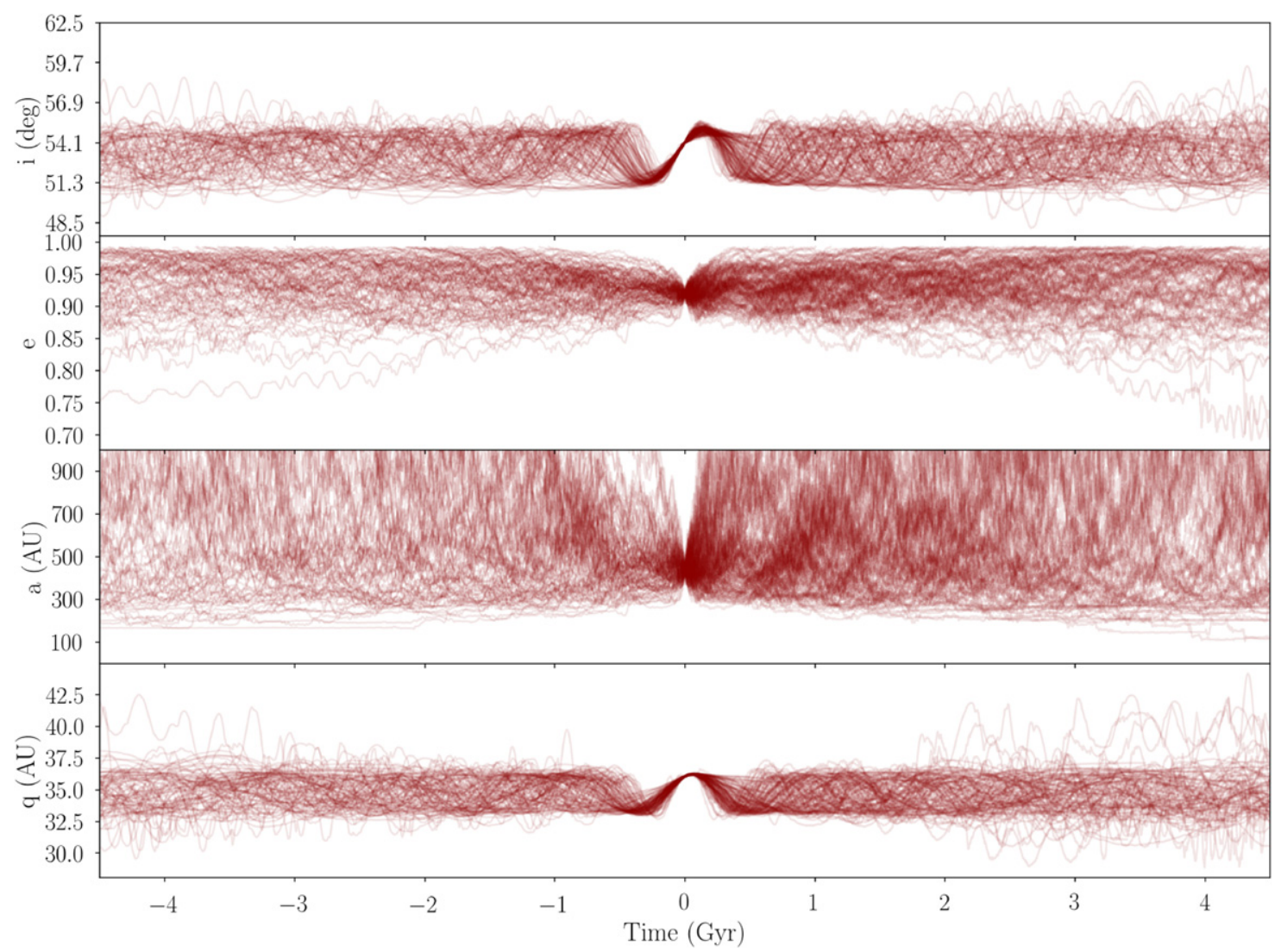

Figure 8. Results of numerical simulations where $2015 \mathrm{BP}_{519}$ is evolved forward and backward in time in the presence of the four giant planets (Set 1; see Table 2). All trials are plotted here; curves that end prematurely before $4.5 \mathrm{Gyr}$ correspond to the integrations where a clone becomes dynamically unstable (collision into the central body, ejection from the system, physical collision with a planet, or a scattering event resulting in an unbound orbit). For trials that remain dynamically stable, the inclination and eccentricity are relatively well constrained to values near their initial conditions. The semimajor axis of $2015 \mathrm{BP}_{519}$ diffuses rapidly.

wide range of values, the corresponding evolution in eccentricity is constrained by the behavior of the perihelion: to leading order, the perihelion distance of $2015 \mathrm{BP}_{519}$ remains well confined. Specifically, $q$ is constant to within $\sim 5$ au over the entire envelope of all dynamically stable clones. The eccentricity evolution of $2015 \mathrm{BP}_{519}$ is thus explained by the requirement that the perihelion remains nearly constant as the semimajor axis varies. Moreover, this behavior is mediated by Neptune. The orbital evolution is consistent with that expected for a member of the scattered disk.

The high present-day inclination of $2015 \mathrm{BP}_{519}$ is more difficult to explain. In the numerical simulations shown in Figure 8 in the context of the currently observed solar system, the orbital inclination of $2015 \mathrm{BP}_{519}$ is found to remain roughly constant. This trend holds for simulations running both backward and forward in time. Since the solar system formed from a disk, we expect the orbital inclination of $2015 \mathrm{BP}_{519}$ to be low at birth. The transition from an initially low inclination orbit to the present-day (high) value must be explained by some mechanism that is not included in our simulations. Some possible explanations include the following: a passing star could excite objects to highly inclined and eccentric orbits; a particularly favorable impact parameter during a close encounter with Neptune could excite an object out of the plane of the solar system; the high inclination could be a fossil from violent migration processes in the early solar system; the self-gravity of a large disk of planetesimals in the scattered disk could excite large mutual inclinations; and finally, the existence of proposed solar system member Planet Nine could lead to secular evolution in eccentricity and inclination for long-period TNOs, thereby producing the current-day orbit of $2015 \mathrm{BP}_{519}$. In this section, we briefly consider the first three possibilities and then examine the Planet Nine hypothesis in detail in Section 5 .

\subsubsection{Scattering Interactions with Other Stars}

As most planetary systems form in clusters (Lada \& Lada 2003; Porras et al. 2003), the solar system is likely to have formed in such an environment (Adams 2010). Dynamical interactions between cluster members can shape the dynamics of the constituent planetary systems (Brasser et al. 2012a). The interactions tend to have a moderate effect (Adams et al. 2006) but can nonetheless sculpt the outer portions of the planetary system or the original disk, which are of interest here. Interactions in the birth cluster are expected to dominate over those that occur later on in the field, but the latter can still be significant. If the trajectory of a binary or single star brings it sufficiently close to a star hosting a planetary system, the geometry of the planetary system can be altered (JiménezTorres et al. 2011). For example, Kenyon \& Bromley (2004) discussed the possibility that Sedna's orbit is the result of a passing star perturbing the orbit of objects in the Kuiper Belt. They found that if such a star had its own disk of planets and planetesimals, then some objects could be captured into our solar system on high-inclination orbits. It is thus possible that $2015 \mathrm{BP}_{519}$ is the result of interactions between our solar system and an external perturbing body. The interaction cross 
sections for such events are much larger at the low flyby speed realized in young embedded clusters ( $\mathrm{Li} \&$ Adams 2015), so the required event is more likely to occur in the birth cluster (compared to the field).

\subsubsection{Scattering Interactions with Neptune}

As discussed in Duncan et al. (1987), TNOs will experience a perturbation in orbital energy at each periapsis, when the TNO passes closest to the orbit of Neptune. Although Figure 8 demonstrates that in our set of backward integrations, 2015 $\mathrm{BP}_{519}$ has retained roughly the same inclination for the past $4.5 \mathrm{Gyr}$, there is some variation among the individual trials. More specifically, one particular integration in the backward time direction attained (at one point) an orbital inclination of $60^{\circ}$, although such a large value was not attained in any of the other integrations in either direction. With a large enough set of simulations, one could find the probability that $2015 \mathrm{BP}_{519}$ could originate in an orbit closer to the plane of the solar system and subsequently evolve into its present orbit. In this scenario, $2015 \mathrm{BP}_{519}$ could have reached its high inclination from a series of extreme scattering events with Neptune. Our current set of numerical simulations shows that this scenario is possible but unlikely.

\subsubsection{Remnant of Planetary Migration}

The Nice model (Gomes et al. 2005; Morbidelli et al. 2005; Tsiganis et al. 2005) suggests that even if the solar system starts as a roughly coplanar disk, the planets attain their small eccentricities and inclinations through scattering events with the large reservoir of planetesimals in the outer solar system. Some of these bodies will be forced to high eccentricities and inclinations, while others will be able to maintain their lower $(e, i)$ distributions (Levison et al. 2008). This scenario is characterized by a short period of extreme instability, which corresponds to the Late Heavy Bombardment inferred in the history of our solar system (at an age of $\sim 600 \mathrm{Myr}$ ). As a result of this violent period, high-inclination objects can be created from objects originating at the outer edge of the planetesimal disk. Although it is unclear how an object with a semimajor axis as high as that of $2015 \mathrm{BP}_{519}$ would be generated in this process, we cannot exclude the idea that $2015 \mathrm{BP}_{519}$ 's currently observed orbital inclination may come from a period of violent instability in the early history of the solar system.

Another explanation for high semimajor axis, high-eccentricity orbits could be the diffusion hypothesis proposed in Bannister et al. (2017) for the generation of 2013 SY Y9 $_{99}$ 's orbit. Objects with the longest orbital periods may sequentially scatter outward, detach their perihelia through galactic tides, and then diffuse inward into orbits with long periods and detached perihelia. Galactic tides start to dominate once an object attains a semimajor axis of roughly $3000 \mathrm{au}$ or more (Duncan et al. 1987), meaning that the currently observed TNOs are not generally susceptible to these effects. This mechanism does appear to describe 2013 SY $_{99}$, an object with a semimajor axis $a \approx 730$ au and an eccentricity of 0.93 , which fits into the dynamical class of objects that would be produced by this mechanism. However, $2015 \mathrm{BP}_{519}$ 's perihelion is not sufficiently detached (35 versus 50 au for $2013 \mathrm{SY}_{99}$ ) for this mechanism to operate.

Another explanation for this object's extreme orbit could be galactic tides acting on remnants of the inner Oort cloud. It has been suggested (Brasser et al. 2012b) that Centaurs may come from the inner Oort cloud rather than the scattered disk. Brasser et al. (2012a) showed that the median inclination of the inner Oort cloud should be around $50^{\circ}$. As mentioned in Brasser et al. (2012a), the number and orbital parameters of objects with large semimajor axes can be used to constrain birth cluster properties. An object at 450 au would be near the inner 2\%-5\% of the cloud, depending on the density profile used. However, objects formed via this mechanism (such as SY99; Bannister et al. 2017) would be expected to have detached perihelia distances, which $2015 \mathrm{BP}_{519}$ does not.

Silsbee \& Tremaine (2018) discussed the possibility that a potentially planetary-mass object (sub-Earth mass) could have formed among the giant planets, and its influence during Neptune's migration could have excited TNOs to present-day high inclinations. This object is distinct from the Planet Nine discussed in the next section.

\subsubsection{Self-gravity of the Scattered Disk}

A sufficiently large (1-10 Earth masses in total mass) eccentric disk would experience an instability due to the selfgravity of the disk (Madigan \& McCourt 2016). This proposed instability could cause clustering in $\omega$ (as observed) for the objects experiencing the instability and a subsequent pumping of inclination for objects that find their apocenter above the orbital plane (Madigan et al. 2018). This would result in the population of high inclinations for eccentric objects. If the scattered disk contains enough mass to cause the instability, $2015 \mathrm{BP}_{519}$ could undergo this mechanism; for this explanation to be feasible, a large number of additional objects in the scattered disk will need to be found, as the early mass of the scattered disk must have been high for this instability to occur.

\section{Dynamics in the Presence of Planet Nine}

Many recent papers have considered the existence of a possible ninth planet. In this section, we consider how the existence of Planet Nine would alter the orbital behavior and evolution of $2015 \mathrm{BP}_{519}$. In considering possible dynamical interactions between $2015 \mathrm{BP}_{519}$ and Planet Nine, there are two main classes of effects that may be relevant.

1. Constant- $a$ evolution (while in or near resonance with another body). Due to $2015 \mathrm{BP}_{519}$ 's large semimajor axis, we do not expect Neptune resonances to be relevant. The longest-period objects known to be in resonance with Neptune have semimajor axes of $\sim 130$ au (Volk et al. 2018). Object $2015 \mathrm{BP}_{519}$ 's semimajor axis of $\sim 450$ au is likely too large for these processes to be relevant. However, resonances with Planet Nine may be important.

2. Diffusion and scattering in $a$ due to close encounters with Neptune or Planet Nine. These encounters may be very close $(<3 \mathrm{au})$ and lead to significant changes in the orbit of $2015 \mathrm{BP}_{519}$ or more distant (5-15 au) and act more as a series of perturbations than an abrupt change.

Both of these modes of evolution can occur over the entire history of the solar system. For example, Figure 9 shows one sample numerical realization of the orbital evolution of 2015 $\mathrm{BP}_{519}$, which demonstrates these two evolutionary modes within a single $4.5 \mathrm{Gyr}$ integration. 


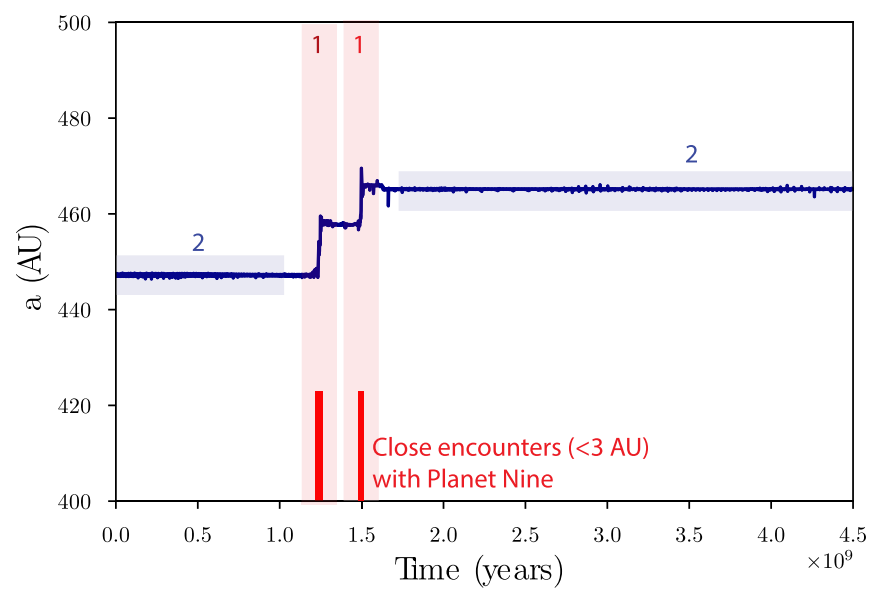

Figure 9. Single clone of $2015 \mathrm{BP}_{519}$ in the presence of Planet Nine drawn from Set 3 of our simulations. There are two modes of evolution, both shown and labeled in this panel. The first (1) occurs when $2015 \mathrm{BP}_{519}$ passes physically close to Planet Nine (a close encounter), and the orbit of the TNO may be slightly jostled. Times when $2015 \mathrm{BP}_{519}$ passes within 3 au of Planet Nine are denoted with red vertical lines. When close encounters occur, the orbit of $2015 \mathrm{BP}_{519}$ is altered and appears to migrate for some time before settling into a new equilibrium semimajor axis. These jumps are the same "resonance hopping" discussed in previous work (Becker et al. 2017). The second mode of evolution shown here (2) occurs when the semimajor axis remains constant but the inclination and eccentricity of $2015 \mathrm{BP}_{519}$ may still evolve. The work in Batygin \& Morbidelli (2017) describes what happens during these (2) regions of constant semimajor axis.

\subsection{Evolution with Constant Semimajor Axis}

The existence of Planet Nine can lead to a behavior in which TNOs "hop" between resonances (Becker et al. 2017; Hadden et al. 2018). This is differentiated from "resonance sticking" (Duncan \& Levison 1997; Robutel \& Laskar 2001; Lykawka \& Mukai 2006), where scattered disk objects are temporarily captured into resonances with Neptune. In the Planet Nine paradigm, TNOs generally spend more time living in resonances than not, with relatively short periods between the attainment of resonances.

An example of what resonance hopping looks like is given in Figure 9, where the semimajor axis makes sudden transitions between relatively long periods at nearly constant values (note that further examples can be found in Figures 8 and 9 of Becker et al. 2017). The transitions in the resonance-hopping paradigm are generally caused by close encounters with either Neptune or Planet Nine.

Batygin \& Morbidelli (2017) conducted a thorough analytic and numerical exploration of the evolution of TNOs in the case where the TNOs remain at a nearly constant semimajor axis. Figure 9 demonstrates the typical behavior of $2015 \mathrm{BP}_{519}$ in the presence of Planet Nine: for extended periods of time, it orbits with a roughly constant semimajor axis, until a close encounter (denoted by red vertical lines in the figure) perturbs the semimajor axis into a different value. A new equilibrium is quickly attained, and the object returns to evolution with a nearly constant semimajor axis $a$. During the long periods of constant- $a$ orbital motion, the dynamics described in Batygin \& Morbidelli (2017) will apply, as described below.

To study the evolution of $2015 \mathrm{BP}_{519}$ under the same conditions starting in the early solar system and integrating to the current day, we conduct another set of simulations (Set 2; see Table 2). In contrast to the earlier Set 1 integrations, where the giant planets were considered as active bodies, these simulations absorb all four gas giants into the quadrupole moment of the central body. The corresponding contribution of the planets to the value of $J_{2}$ is given by

$$
J_{2}=\frac{1}{2} \sum_{j=1}^{4} \frac{m_{j} a_{j}^{2}}{M R_{\mathrm{abs}}^{2}},
$$

where $R_{\text {abs }}$ is the absorbing radius within which objects are removed from the simulation, the index $j$ counts through the four gas giants, $m_{j}$ and $a_{j}$ denote planetary masses and semimajor axes, and $M$ denotes the mass of the central body. This approximation minimizes perturbations in $a$ space, allowing for an easier study of the orbital evolution at constant $a$. As was done in Batygin \& Morbidelli (2017), we initialize the inclination of $2015 \mathrm{BP}_{519}$ to be drawn from a half-normal distribution with mean $0^{\circ}$ and width $5^{\circ}$, which simulates the expected initial conditions in the early solar system. We also include Planet Nine using the best-fit values of its orbital elements ( $a=700 \mathrm{au}, e=0.6, i=20, \omega=150, \Omega=90$ ), which come from Batygin \& Morbidelli (2017) and Millholland \& Laughlin (2017). We also run an additional set of simulations (Set 3) with identical parameters but using the observed inclination of $2015 \mathrm{BP}_{519}$ as drawn from the observationally derived covariance matrix. Simulation Set 2 is intended to study the behavior of an object like $2015 \mathrm{BP}_{519}$ but starting from early in solar system history, before the inclination of $2015 \mathrm{BP}_{519}$ is perturbed to its current-day value. Simulation Set 2 is intended to answer the following question: assuming that $2015 \mathrm{BP}_{519}$ started in the same plane as the outer solar system objects that were present in the early solar system, can secular interactions with Planet Nine excite $2015 \mathrm{BP}_{519}$ 's inclination to its current-day value? For comparison, simulation Set 3 studies the behavior of $2015 \mathrm{BP}_{519}$ from the current day forward (but using the same approximations that are used in Set 2; namely, neglecting perturbations caused by scattering interactions with the giant planets and treating evolution as occurring at constant $a$ ).

In Figure 10, we plot the action-angle evolution of the results of Set 2 using angle

$$
\theta=\Delta \varpi=2 \Omega-\varpi-\varpi_{9}
$$

and coordinate action

$$
\Theta=\frac{\sqrt{1-e^{2}}}{2}(1-\cos i),
$$

as done in Batygin \& Morbidelli (2017).

The resulting evolution of $2015 \mathrm{BP}_{519}$ in this action-angle phase space is plotted in Figure 10. The lines trace the $4.5 \mathrm{Gyr}$ evolution of the realizations of $2015 \mathrm{BP}_{519}$ from simulation Set 2. The star symbol marks the present-day location of 2015 $\mathrm{BP}_{519}$ in this parameter space using its observed inclination, eccentricity, and (expected) $\Delta \varpi$. It is important to note that the remarkably high observed inclination of $2015 \mathrm{BP}_{519}$ is not a guaranteed outcome of these simulations. Nonetheless, the star symbol lies along the teal contours, which describe regions of the phase space to which an initially coplanar $2015 \mathrm{BP}_{519}$ could evolve. These simulations demonstrate that in the case where $2015 \mathrm{BP}_{519}$ starts its life close to the plane containing the solar system planets, it is able to attain its current-day inclination, 


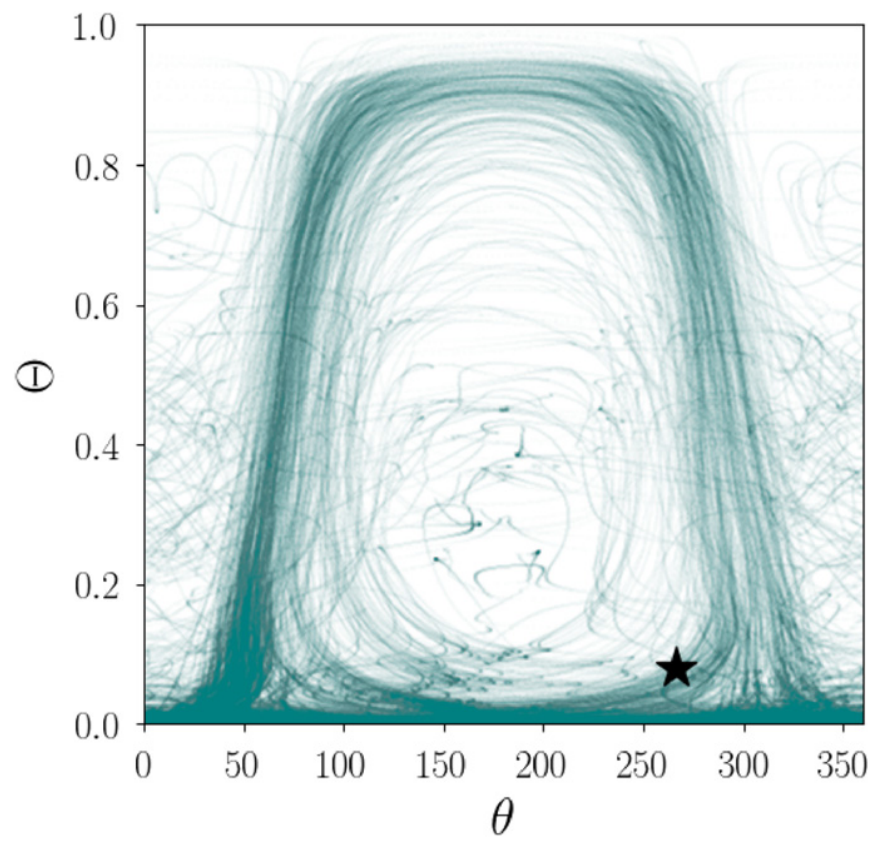

Figure 10. Orbital evolution of $2015 \mathrm{BP}_{519}$ in action-angle space. This figure shows results from simulations where the initial inclination of $2015 \mathrm{BP}_{519}$ was drawn from a half-normal distribution centered at $0^{\circ}$ with a width of $5^{\circ}$ (Set 2; see Table 2). This plot should be compared to the bottom panel of Figure 11 in Batygin \& Morbidelli (2017). The currently observed action-angle coordinates $\theta$ and $\Theta$ (computed using the simulated version of Planet Nine) are marked by the star symbol. The current-day orbital elements of $2015 \mathrm{BP}_{519}$ are easily reproduced in the scenario with Planet Nine and with $2015 \mathrm{BP}_{519}$ starting in the plane with the other solar system objects.

eccentricity, and orbital orientation through secular interactions with Planet Nine alone.

As a result, Set 2 of our simulations shows that orbital evolution with constant semimajor axis $(a)$ evolution can explain how $2015 \mathrm{BP}_{519}$ achieves its observed inclination in the presence of Planet Nine. In other words, the existence of Planet Nine is sufficient to explain the currently observed orbit of $2015 \mathrm{BP}_{519}$.

\subsection{Orbital Evolution with Planet Nine and Neptune}

The constant- $a$ evolution is relevant for the majority of the lifetimes of the TNOs in the presence of Planet Nine, and the behavior of the TNOs will generally be as described in the previous section during those times. Close encounters with Planet Nine do occur even in the idealized simulation Set 2, but they are rare and tend to lead to only small hops between nearby resonances with Planet Nine. However, as the current perihelion distance of $2015 \mathrm{BP}_{519}$ brings it fairly close to the orbit of Neptune during each perihelion passage, the true evolution of $2015 \mathrm{BP}_{519}$ will be affected heavily by those Neptune-2015 $\mathrm{BP}_{519}$ interactions. In Figure 9, we show a sample orbital evolution of $2015 \mathrm{BP}_{519}$ without Neptune. During a close encounter with Planet Nine, 2015 BP $_{519}$ 's orbit is rapidly altered, where the average distance of its orbit diffuses until it is trapped into or near a new resonance. The inclusion of Neptune as an active body increases the number of close encounters experienced by $2015 \mathrm{BP}_{519}$, as it will interact with both Planet Nine and Neptune. This increase in interactions, in turn, allows for the orbit of $2015 \mathrm{BP}_{519}$ to become more heavily perturbed over time.
To test the effect of these kicks from Neptune, we set up another set of simulations (Set 4; see Table 2). In this case, we replace Jupiter, Saturn, and Uranus with an effective $J_{2}$ term to represent the potential of those three planets. However, this time we include Neptune as an active body, which allows Neptune scattering events to be resolved. As before, the energy is conserved to one part in $10^{9}$, and the hybrid symplectic-B-S integrator is used. The other parameters of this set of simulations are summarized in Table 2, and the results are plotted in Figure 11. As expected, in this new set of simulations, $2015 \mathrm{BP}_{519}$ appears to be significantly less dynamically stable than in the previous sets (which do not include an active Neptune). However, part of this apparent dynamical instability is due to the nonphysical absorbing radius used in the simulations: specifically, we remove particles from the simulation when they reach orbital radii within the absorbing radius. This inner boundary is set to be $9.8 \mathrm{au}$ in this case, since we are replacing the Sun and inner three giant planets with an oblate central body with a larger radius to represent the effective quadrupole term of the entire system.

As a result of the complication outlined above, ensembles of simulations that use effective $J_{2}$ terms (like Set 4) to represent time-averaged planetary orbits cannot be used to study the final outcomes of these objects. For example, if the orbit of a realization of $2015 \mathrm{BP}_{519}$ was to evolve to the point where the clone becomes a Jupiter-family comet, simulation Set 4 would not resolve this end state and would instead classify the clone as dynamically unstable. On the other hand, this approximation can be used to describe the expected secular evolution for objects that remain a part of the same dynamical population.

\subsection{Orbital Evolution with Planet Nine and the Four Giant Planets}

Both of the previous sections discussing the orbital evolution of $2015 \mathrm{BP}_{519}$ in the presence of Planet Nine replaced some (or all) of the gas giants with an effective $J_{2}$ term. This timesaving integration strategy has been used extensively in the Planet Nine literature (Batygin \& Brown 2016a; Brown \& Batygin 2016; Hadden et al. 2018; Millholland \& Laughlin 2017). In Section 5.2, we showed that the physical presence of Neptune leads to a greater number of transitions ("hops") between Planet Nine (true or near) resonances. Next, our final set of simulations (Set 5) investigates the effect of including all four gas giants as active bodies. The details of Set 5 are given in Table 2. One important detail about this set of simulations is that since all the gas giants are included as active particles (and terrestrial planets ignored), no planets need to be modeled as perturbations on the solar $J_{2}$. As such, the absorbing radius of the central body is set equal to the solar radius. This aspect of the simulations allows for the resolution of outcomes where $2015 \mathrm{BP}_{519}$ settles into a stable orbit with a perihelion distance that passes into the inner solar system; the results of this set of simulations are shown in Figure 12. This figure appears very similar to Figure 11 but describes the full motion of 2015 $\mathrm{BP}_{519}$. The striking similarity between the two figures can be used as a justification for using the $J_{2}$ approximation when secular evolution is being studied.

\section{Discussion}

In this work, we present the discovery and dynamical analysis of a new ETNO, a population defined as those objects 


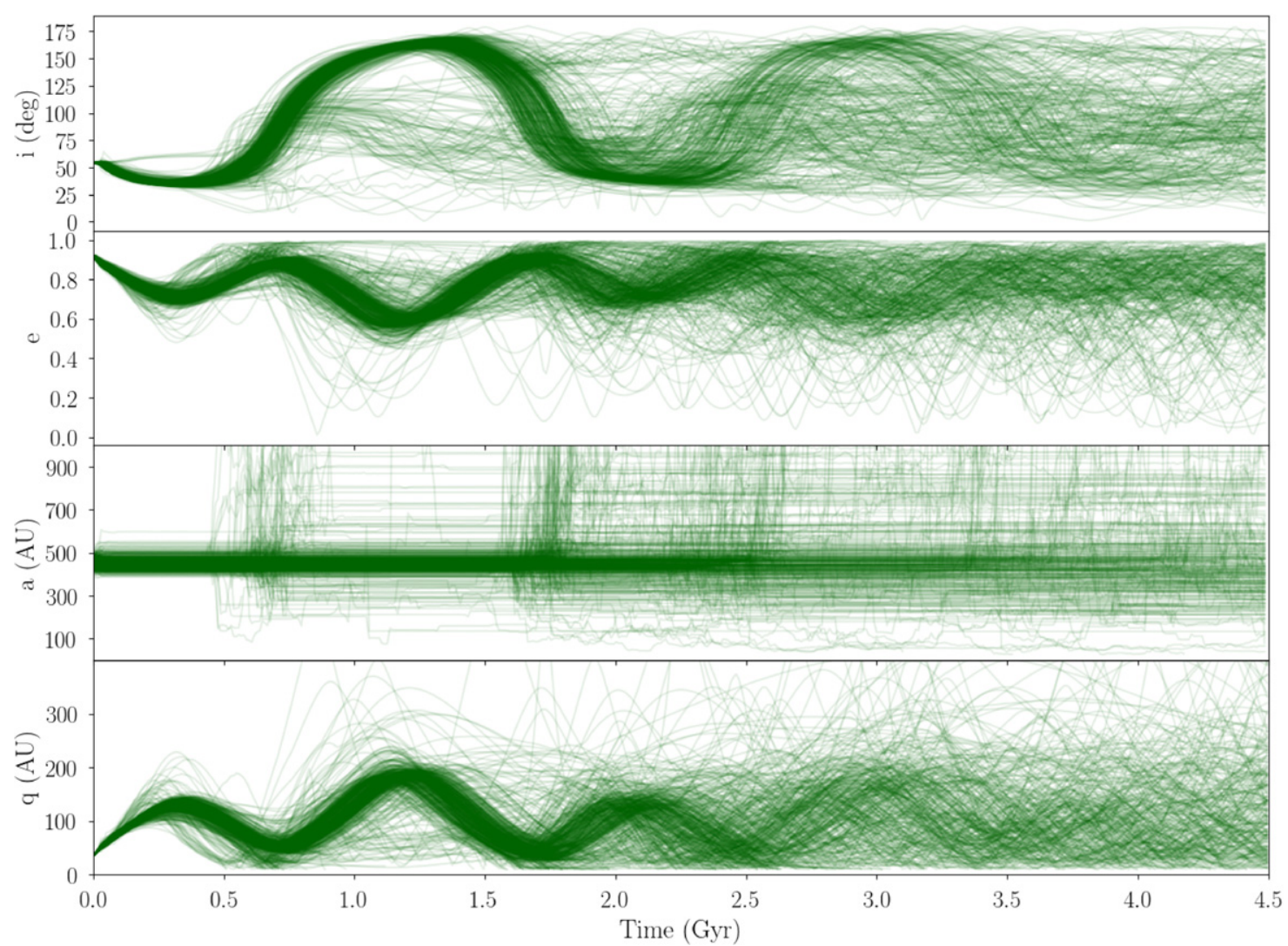

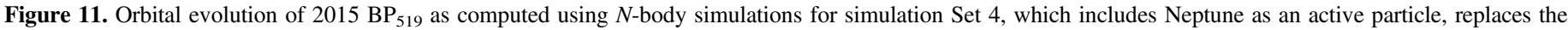

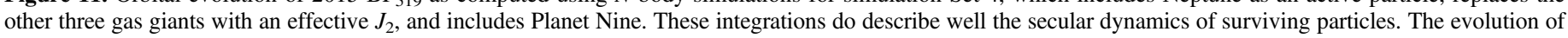
the semimajor axis also shows the horizontal banding structure in the semimajor axis, which is characteristic of resonance hopping.

with $a>250$ and $q>30$ au. Because $2015 \mathrm{BP}_{519}$ has the largest eccentricity and inclination of any of the ETNOs, it allows us to probe the behavior of a new regime in the solar system. Ideally, DES and other surveys will find more of these high-inclination, large semimajor axis objects. Once such a population is found and grows to a sufficient size, it will inform a variety of hypotheses about the structure of the outer solar system and the migration of the giant planets. For now, while the number of such known objects is small, we have performed an in-depth study of the dynamical evolution of $2015 \mathrm{BP}_{519}$ in various scenarios with two goals: first, we would like to make whatever insights are possible with a single object to improve our understanding of the outer solar system; second, we would like to determine which hypotheses and analyses will be most fruitful for future study once more of these objects are found.

Our analysis of the orbital evolution of $2015 \mathrm{BP}_{519}$ using forward and backward integrations has revealed that it is difficult to reproduce $2015 \mathrm{BP}_{519}$ 's high current-day inclination in the known solar system without considering some other mechanism. In simulation Set 1, which studied the evolution of this object in the known solar system, zero out of the 260 simulated clones of $2015 \mathrm{BP}_{519}$ attained inclinations less than $48^{\circ}$ or greater than $60^{\circ}$ when integrations were initialized with $2015 \mathrm{BP}_{519}$ 's measured inclination of $\sim 54^{\circ}$. This strong confinement in inclination space that is evident in the numerical simulations requires us to consider other mechanisms to excite the inclination of this object. Some potential explanations (discussed in Section 4.2) include a stellar flyby, a remnant excitation from the early migration of the giant planets, a particularly serendipitous outcome not captured by our 260
$N$-body simulations, or an inclination instability caused by the self-gravity of a massive scattered disk.

One additional explanation to those listed above is the existence of a ninth planet in our solar system, as proposed by Trujillo \& Sheppard (2014) and Batygin \& Brown (2016a). As shown in Figure 10, in the presence of Planet Nine, $2015 \mathrm{BP}_{519}$ can start out with a relatively low inclination and easily attain its current-day inclination. Additionally, as shown in Figure 13, $2015 \mathrm{BP}_{519}$ 's orbital angles $\omega, \Omega$, and $\varpi$ appear to be consistent with the clustering first noted in Trujillo \& Sheppard (2014). This clustering in physical space has been proposed to be caused by the $\sim 10$ Earth mass Planet Nine at 700 au (Batygin \& Brown 2016a) and is the line of evidence most commonly used to support the existence of Planet Nine. Although 2015 $\mathrm{BP}_{519}$ does appear to fit into this paradigm, the physicality of the clustering remains a contentious piece of evidence for Planet Nine (Brown 2017; Shankman et al. 2017a). In Figure 5, we showed the bias in DES detections of objects with varying orbital angles $\omega$ and $\Omega$ but the same $(a, e, i)$ as $2015 \mathrm{BP}_{519}$. For $2015 \mathrm{BP}_{519}$, at least, the biases are sufficiently mild that it seems that $2015 \mathrm{BP}_{519}$ can be used as evidence of the existence of the clustering. However, the observational biases we determine for $2015 \mathrm{BP}_{519}$ do not tell us anything about other objects that may be found by DES or other surveys: without fully accounting for the observational biases for each individual survey that has discovered these ETNOs, it cannot fully be determined how much of the clustering is physical and how much is due to observational bias. Past surveys have been able to quantify this: the Deep Ecliptic Survey had well-documented pointings and, as a result, was able to construct a model of its 


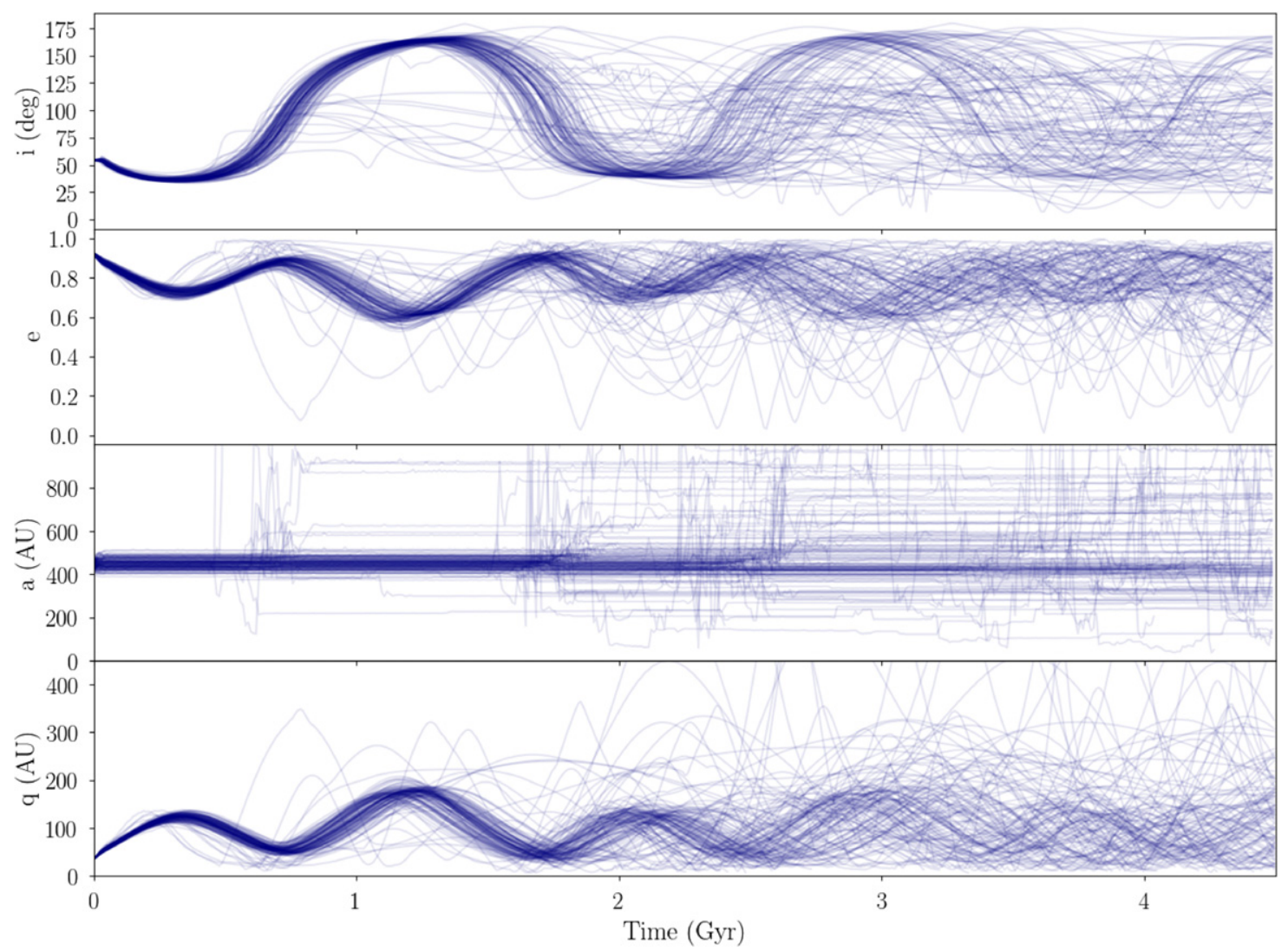

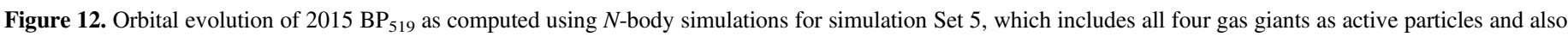

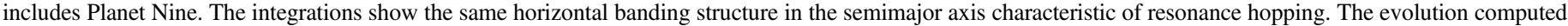
here is very similar in secular trajectory to that of Set 4 (Figure 11).

detection biases (Adams et al. 2014). Similarly, the Outer Solar Systems Origin Survey has quantified its own biases (Lawler et al. 2018). Future work (S. J. Hamilton et al. 2018, in preparation) will do the same for the DES and enable a better understanding of whether the clustering suggesting Planet Nine's existence is real or a sampling bias.

However, $2015 \mathrm{BP}_{519}$ does provide additional diagnostics unrelated to angular clustering that inform the Planet Nine debate. Batygin \& Brown (2016a) predicted that highinclination KBOs would serve as an important constraint on Planet Nine's properties. Subsequently, dynamical analysis presented in Batygin \& Morbidelli (2017) suggests that the population of highly inclined Centaurs can be explained by the presence of Planet Nine. Shankman et al. (2017b) predicted that if there is a ninth planet in the solar system, there should also be a reservoir of high- $i$ TNOs that exhibit clustering of their orbits with the existing population. Finally, Batygin \& Morbidelli (2017) provided a model of the secular evolution expected for high- $i$, high- $a$ objects but were only able to test it on objects with $q<30 \mathrm{au}$. Object $2015 \mathrm{BP}_{519}$ is the first known high- $a$ $(a>250 \mathrm{au})$, high- $i\left(i>40^{\circ}\right)$, high- $q(q>30$ au) object, a class of objects whose existence was predicted by Batygin \& Morbidelli (2017). It is the first discovered high- $i$ object, and it fits into the Planet Nine paradigm as predicted by this previous work.

In Figure 14, we show the fraction of surviving objects that have retrograde orbits for three of the different simulation sets used in this work. A sizable fraction of $2015 \mathrm{BP}_{519}$ 's potential future orbits attain retrograde orientations, an outcome predicted in Batygin \& Brown (2016b) and Batygin \& Morbidelli (2017). A subset of these also evolve to lower semimajor axes, potentially resulting in $2015 \mathrm{BP}_{519}$ eventually becoming a retrograde Centaur; however, our simulations show that it is more likely that $2015 \mathrm{BP}_{519}$ retains a large semimajor axis and retrograde configuration than that it migrates inward and becomes a Centaur. In the presence of Planet Nine, TNOs with orbits as extreme as that of $2015 \mathrm{BP}_{519}$ would appear to cycle though populations, changing their orbital inclinations and perihelion distances rather than living at roughly constant perihelion distances (as they would in the known solar system without Planet Nine; see Figure 8). Finally, the presence of Planet Nine in the solar system naturally produces objects with orbits like that of $2015 \mathrm{BP}_{519}$, a feature that cannot be reproduced in the solar system without Planet Nine without invoking some other mechanism (such as interaction with a passing star or a 1-10 Earth mass scattered disk that can cause an inclination instability; Madigan \& McCourt 2016; Madigan et al. 2018).

Although $2015 \mathrm{BP}_{519}$ appears to fit well into the Planet Nine paradigm and aid in a better differentiation between these two potential scenarios - a solar system with or without Planet Nine -more objects of this type need to be found. Future work using the DES will both identify additional high semimajor axis, high-inclination objects that will help us better understand the high-inclination structure of the outermost regions of the solar system and make a more definitive statement on the existence of Planet Nine. 


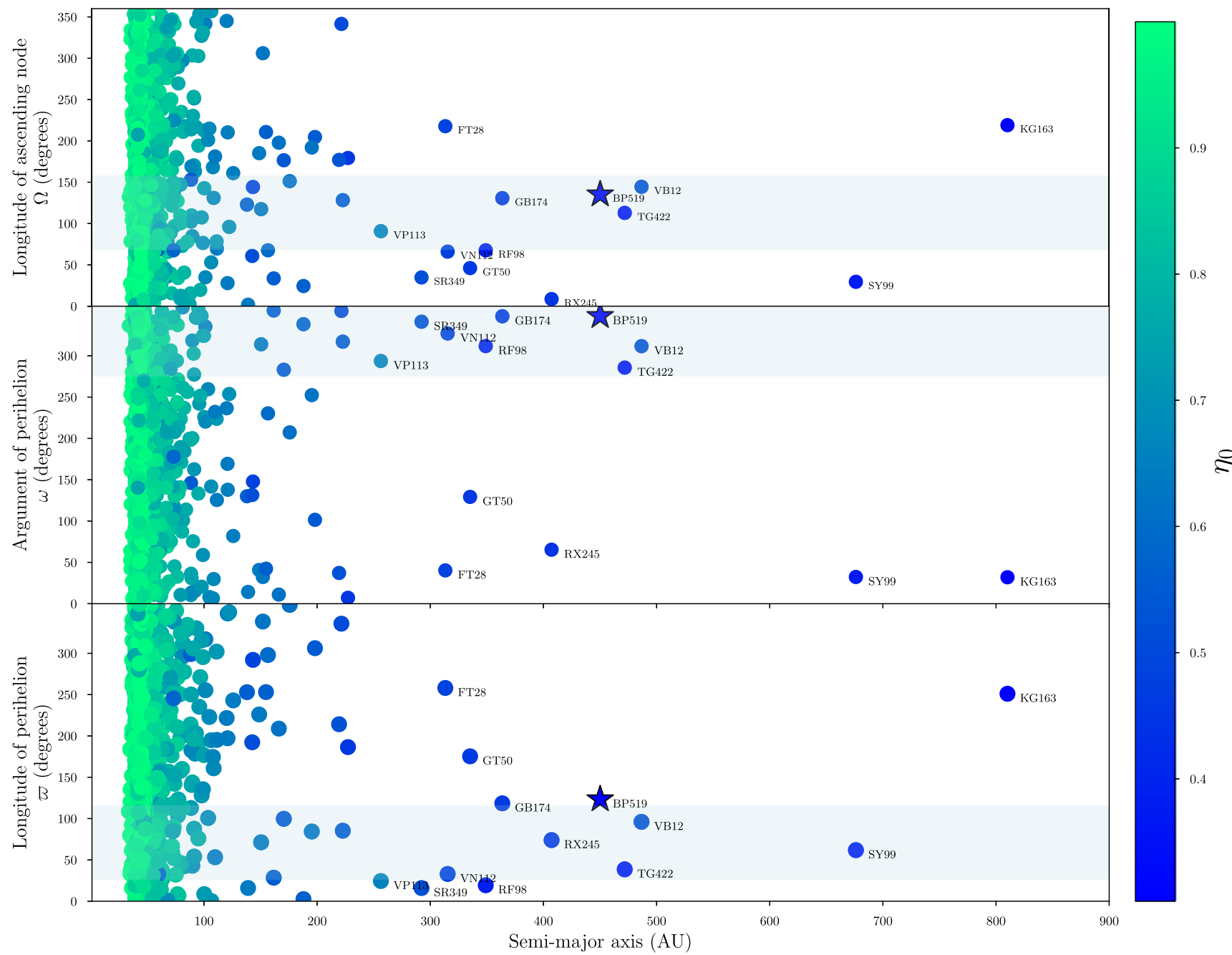

Figure 13. Visualization of the two orbital angles $\Omega$ (longitude of ascending node; top panel) and $\omega$ (argument of perihelion; middle panel), along with their sum longitude of perihelion $\varpi=\omega+\Omega$ (bottom panel). The points are color-coded by the specific angular momentum of the orbit $\eta_{0}=\sqrt{1-e^{2}}$ cos $i$. The plot includes all objects with $q>30$ au and data quality $U<6$ from the MPC database (Marsden et al. 1978), with $2015 \mathrm{BP}_{519}$ denoted as a star. Horizontal bars denote the approximate regions of clustering in each angle, as identified in Batygin \& Brown (2016a).

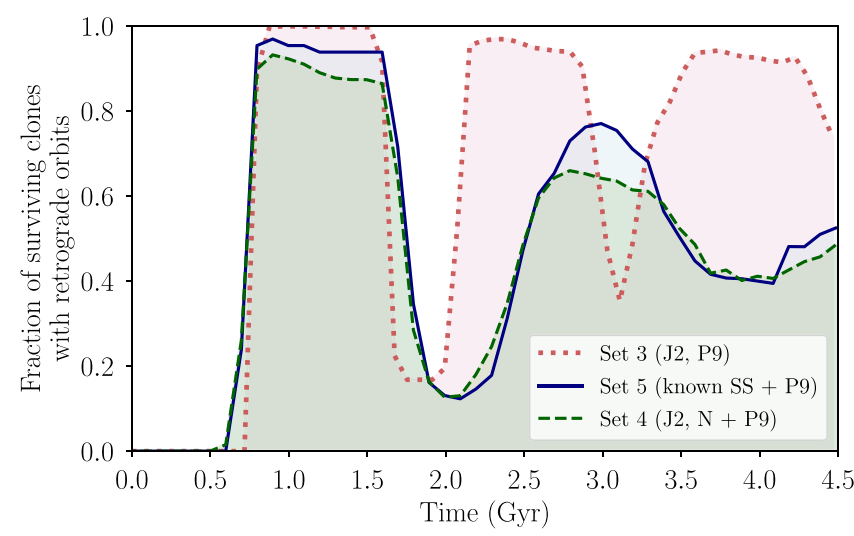

Figure 14. Measure of the fraction of $2015 \mathrm{BP}_{519}$ clones that attain retrograde orbits as a function of time in the numerical simulations that include Planet Nine. The coherence between all sets of simulations is due to a single realization of Planet Nine's orbital elements being used for all simulations. The good agreement between the simulations that used a $J_{2}$ approximation (Set 4) and those that included all gas giants as active particles (Set 5) suggests that the $J_{2}$ approximation (while keeping Neptune an active particle) is appropriate for studying the orbital evolution of surviving particles, even if it does not work well on its own for studying the dynamical stability. The integrations in Set 1 (which included the known solar system and no Planet Nine) never attain retrograde geometries.

\section{Conclusion}

This paper reports the detection and initial dynamical analysis of the ETNO $2015 \mathrm{BP}_{519}$. This object was discovered as part of the DES and adds to the growing inventory of unusual bodies in the outer solar system. Our main results can be summarized as follows.

(1) The estimated orbital elements for this new (minor) member of the solar system include semimajor axis $a \approx 450$ au, eccentricity $e \approx 0.92$, and inclination $i \approx 54^{\circ}$. With these orbital properties, $2015 \mathrm{BP}_{519}$ resides well outside the classical Kuiper Belt. On the other hand, the perihelion distance is only $q \sim 36 \mathrm{au}$, close enough to be influenced by Neptune.

(2) The newly discovered body $2015 \mathrm{BP}_{519}$ is the most extreme of the ETNOs found to date. This claim can be quantified using the reduced Kozai action $\eta_{0}$ (see Equation (5)), which is equivalent to the $z$-component of the specific orbital angular momentum. Among all known solar system objects, $2015 \mathrm{BP}_{519}$ has the most extreme value of this parameter, as shown in Figure 7.

(3) Object $2015 \mathrm{BP}_{519}$ provides support for the Planet Nine hypothesis. If the object is formed in the plane of the solar system, as expected, then there is a low probability that its orbit 
can attain the observed high inclination through dynamical processes involving only the known planets. In contrast, the observed orbital elements of $2015 \mathrm{BP}_{519}$ are readily produced through dynamical interactions if the solar system also contains Planet Nine (see Figure 10).

This material is based upon work supported by the National Aeronautics and Space Administration under grant No. NNX17AF21G issued through the SSO Planetary Astronomy Program and by NSF grant AST-1515015. We would like to thank Andrew Vanderburg, Ellen Price, Linn Eriksson, Melaine Saillenfest, and Mike Brown for many useful conversations. We would like to thank Michele Bannister for useful discussions and methods advice. We would like to thank Konstantin Batygin for his careful review of the manuscript and suggestions that greatly improved this work. We thank Marty Kandes and Mats Rynge for help running simulations on Open Science Grid's high-throughput computing resources (operated through XSEDE). JCB, SJH, and LM are supported by NSF Graduate Research Fellowship Grant No. DGE 1256260. This work used the Extreme Science and Engineering Discovery Environment (XSEDE), which is supported by National Science Foundation grant number ACI-1053575. This research was done using resources provided by the Open Science Grid, which is supported by the National Science Foundation and the U.S. Department of Energy's Office of Science through allocations TG-AST150033 and TG-AST170008. This research has made use of data and services provided by the International Astronomical Union's Minor Planet Center.

Funding for the DES Projects has been provided by the U.S. Department of Energy, the U.S. National Science Foundation, the Ministry of Science and Education of Spain, the Science and Technology Facilities Council of the United Kingdom, the Higher Education Funding Council for England, the National Center for Supercomputing Applications at the University of Illinois at Urbana-Champaign, the Kavli Institute of Cosmological Physics at the University of Chicago, the Center for Cosmology and Astro-Particle Physics at the Ohio State University, the Mitchell Institute for Fundamental Physics and Astronomy at Texas A\&M University, Financiadora de Estudos e Projetos, Fundação Carlos Chagas Filho de Amparo à Pesquisa do Estado do Rio de Janeiro, Conselho Nacional de Desenvolvimento Científico e Tecnológico and the Ministério da Ciência, Tecnologia e Inovação, the Deutsche Forschungsgemeinschaft, and the Collaborating Institutions in the Dark Energy Survey.

The Collaborating Institutions are Argonne National Laboratory, the University of California at Santa Cruz, the University of Cambridge, Centro de Investigaciones Energéticas, Medioambientales y Tecnológicas-Madrid, the University of Chicago, University College London, the DES-Brazil Consortium, the University of Edinburgh, the Eidgenössische Technische Hochschule (ETH) Zürich, Fermi National Accelerator Laboratory, the University of Illinois at UrbanaChampaign, the Institut de Ciències de l'Espai (IEEC/CSIC), the Institut de Física d'Altes Energies, Lawrence Berkeley National Laboratory, the Ludwig-Maximilians Universität München and the associated Excellence Cluster Universe, the University of Michigan, the National Optical Astronomy Observatory, the University of Nottingham, The Ohio State University, the University of Pennsylvania, the University of Portsmouth, SLAC National Accelerator Laboratory, Stanford University, the University of Sussex, Texas A\&M University, and the OzDES Membership Consortium.

Based in part on observations at Cerro Tololo InterAmerican Observatory, National Optical Astronomy Observatory, which is operated by the Association of Universities for Research in Astronomy (AURA) under a cooperative agreement with the National Science Foundation.

The DES data management system is supported by the National Science Foundation under grant Numbers AST1138766 and AST-1536171. The DES participants from Spanish institutions are partially supported by MINECO under grants AYA2015-71825, ESP2015-66861, FPA2015-68048, SEV-2016-0588, SEV-2016-0597, and MDM-2015-0509, some of which include ERDF funds from the European Union. IFAE is partially funded by the CERCA program of the Generalitat de Catalunya. Research leading to these results has received funding from the European Research Council under the European Union's Seventh Framework Program (FP7/ 2007-2013), including ERC grant agreements 240672, 291329, and 306478. We acknowledge support from the Australian Research Council Centre of Excellence for All-sky Astrophysics (CAASTRO) through project number CE110001020 and the Brazilian Instituto Nacional de Ciência e Tecnologia (INCT) e-Universe (CNPq grant 465376/2014-2).

This manuscript has been authored by the Fermi Research Alliance, LLC, under contract No. DE-AC02-07CH11359 with the U.S. Department of Energy, Office of Science, Office of High Energy Physics. The United States Government retains and the publisher, by accepting the article for publication, acknowledges that the United States Government retains a nonexclusive, paid-up, irrevocable, worldwide license to publish or reproduce the published form of this manuscript, or allow others to do so, for United States Government purposes.

Software: WCSfit (Bernstein et al. 2017), mp_ephem (https://github.com/OSSOS/liborbfit), pandas (McKinney et al. 2010), IPython (Pérez \& Granger 2007), matplotlib (Hunter 2007), scipy (Jones et al. 2001), numpy (Oliphant 2006), Jupyter (Kluyver et al. 2016).

\section{Appendix ETNO Data Table}

In Table 3, we list the previously known TNOs with semimajor axis values greater than 250 au and present their best-fit orbits using currently available data. $2015 \mathrm{BP}_{519}$ is the newest member of this population. 
Table 3

Orbital Elements of ETNOs

\begin{tabular}{|c|c|c|c|c|c|c|}
\hline Object & $a(\mathrm{au})$ & $e$ & $i$ & $\omega$ & $\Omega$ & $H$ (abs. mag) \\
\hline $2003 \mathrm{VB}_{12}$ & $507 \pm 10$ & $0.8496 \pm 0.003$ & $11.9 \pm 0.1$ & $311.3 \pm 0.1$ & $144.4 \pm 0.1$ & 1.5 \\
\hline $2007 \mathrm{TG}_{422}$ & $503 \pm 0.35$ & $0.93 \pm 0.001$ & $18.6 \pm 0.1$ & $285.7 \pm 0.1$ & $112.9 \pm 0.1$ & 6.2 \\
\hline $2010 \mathrm{~GB}_{174}$ & $351 \pm 9$ & $0.862 \pm 0.004$ & $21.6 \pm 0.1$ & $347.2 \pm 0.1$ & $130.7 \pm 0.1$ & 6.6 \\
\hline $2013 \mathrm{FT}_{28}$ & $295 \pm 7$ & $0.853 \pm 0.004$ & $17.4 \pm 0.1$ & $40.7 \pm 0.1$ & $217.7 \pm 0.1$ & 6.7 \\
\hline $2013 \mathrm{RF}_{98}$ & $363 \pm 5$ & $0.9 \pm 0.001$ & $29.6 \pm 0.1$ & $311.8 \pm 0.1$ & $67.6 \pm 0.1$ & 8.7 \\
\hline $2013 \mathrm{SY}_{99}$ & $735 \pm 15$ & $0.932 \pm 0.007$ & $4.2 \pm 0.1$ & $32.2 \pm 0.1$ & $29.5 \pm 0.1$ & 6.8 \\
\hline $2015 \mathrm{KG}_{163}$ & $680 \pm 2$ & $0.94 \pm 0.001$ & $14 \pm 0.1$ & $32.1 \pm 0.1$ & $219.1 \pm 0.1$ & 8.1 \\
\hline $2015 \mathrm{RX}_{245}$ & $430 \pm 20$ & $0.894 \pm 0.001$ & $12.1 \pm 0.1$ & $65.2 \pm 0.1$ & $8.6 \pm 0.1$ & 6.1 \\
\hline $2004 \mathrm{VN}_{112}$ & $316 \pm 1$ & $0.8505 \pm 0.0005$ & $25.6 \pm 0.1$ & $327.1 \pm 0.1$ & $66 \pm 0.1$ & 6.5 \\
\hline $2014 \mathrm{FE}_{72}$ & $1655 \pm 336$ & $0.98 \pm 0.02$ & $20.64 \pm 0.1$ & $133.89 \pm 0.04$ & $336.84 \pm 0.1$ & 6.1 \\
\hline
\end{tabular}

Note. Barycentric osculating elements for the currently known set of TNOs with $a>250$ and $q>30$ au. Excluding our new object 2015 BP519, solutions were drawn from Shankman et al. (2017a) and Bannister et al. (2017) for all objects except 2013 RF98, 2007 TG422, and 2014 FE72. The barycentric orbital solutions for these three objects were fit using the OSSOS (Bannister et al. 2016) implementation (available at https://github.com/OSSOS/liborbfit and from the Python Package Index via pip install mp_ephem) of the Bernstein \& Khushalani (2000) orbit fitter using the observations of each object available (https://www. minorplanetcenter.net/db_search/ as of 2/1/2018) at the Minor Planet Center.

\section{ORCID iDs}

J. C. Becker (1) https://orcid.org/0000-0002-7733-4522

T. Khain (1) https://orcid.org/0000-0001-7721-6457

S. J. Hamilton (1) https://orcid.org/0000-0002-6126-8487

F. C. Adams (i) https://orcid.org/0000-0002-8167-1767

D. W. Gerdes (i) https://orcid.org/0000-0001-6942-2736

S. Millholland (1) https://orcid.org/0000-0003-3130-2282

Hsing Wen Lin (林省文) (1) https://orcid.org/0000-00017737-6784

J. Annis (1) https://orcid.org/0000-0002-0609-3987

D. Brooks 니 https://orcid.org/0000-0002-8458-5047

J. García-Bellido (i) https://orcid.org/0000-0002-9370-8360

R. A. Gruendl (1) https://orcid.org/0000-0002-4588-6517

K. Kuehn (1) https://orcid.org/0000-0003-0120-0808

J. L. Marshall (1) https://orcid.org/0000-0003-0710-9474

R. Miquel (ㄷ) https://orcid.org/0000-0002-6610-4836

A. A. Plazas (i) https://orcid.org/0000-0002-2598-0514

E. Sanchez (i) https://orcid.org/0000-0002-9646-8198

M. Smith (1) https://orcid.org/0000-0002-3321-1432

M. Soares-Santos (10) https://orcid.org/0000-0001-6082-8529

M. E. C. Swanson (i) https://orcid.org/0000-0002-1488-8552

A. R. Walker (i) https://orcid.org/0000-0002-7123-8943

\section{References}

Adams, E. R., Gulbis, A. A. S., Elliot, J. L., et al. 2014, AJ, 148, 55 Adams, F. C. 2010, ARA\&A, 48, 47

Adams, F. C., Proszkow, E. M., Fatuzzo, M., \& Myers, P. C. 2006, ApJ, 641, 504

Ahn, C. P., Alexandroff, R., Allende Prieto, C., et al. 2014, ApJS, 211, 17

Bailey, E., Batygin, K., \& Brown, M. E. 2016, AJ, 152, 126

Bannister, M. T., Kavelaars, J. J., Petit, J.-M., et al. 2016, AJ, 152, 70

Bannister, M. T., Shankman, C., Volk, K., et al. 2017, AJ, 153, 262

Batygin, K., \& Brown, M. E. 2016a, AJ, 151, 22

Batygin, K., \& Brown, M. E. 2016b, ApJL, 833, L3

Batygin, K., \& Morbidelli, A. 2017, AJ, 154, 229

Becker, J. C., Adams, F. C., Khain, T., Hamilton, S. J., \& Gerdes, D. 2017, AJ, 154,61

Bernstein, G., \& Khushalani, B. 2000, AJ, 120, 3323

Bernstein, G. M., Armstrong, R., Plazas, A. A., et al. 2017, PASP, 129, 074503

Bernstein, J. P., Kessler, R., Kuhlmann, S., et al. 2012, ApJ, 753, 152

Brasser, R., Duncan, M. J., Levison, H. F., Schwamb, M. E., \& Brown, M. E. 2012a, Icar, 217, 1
Brasser, R., Schwamb, M. E., Lykawka, P. S., \& Gomes, R. S. 2012b, MNRAS, 420, 3396

Bromley, B. C., \& Kenyon, S. J. 2016, ApJ, 826, 64

Brown, M. E. 2017, AJ, 154, 65

Brown, M. E., \& Batygin, K. 2016, ApJL, 824, L23

Brown, M. E., Trujillo, C., \& Rabinowitz, D. 2004, ApJ, 617, 645

Brucker, M. J., Grundy, W. M., Stansberry, J. A., et al. 2009, Icar, 201, 284

Chambers, J. E. 1999, MNRAS, 304, 793

Chen, Y.-T., Kavelaars, J. J., Gwyn, S., et al. 2013, ApJL, 775, L8

Chen, Y.-T., Lin, H. W., Holman, M. J., et al. 2016, ApJL, 827, L24

Dark Energy Survey Collaboration, Abbott, T., Abdalla, F. B., et al. 2016, MNRAS, 460, 1270

Chen, Y.-T., Lin, H. W., Holman, M. J., et al. 2016, ApJL, 827, L24

de la Fuente Marcos, C., \& de la Fuente Marcos, R. 2016, MNRAS, 462, 1972

Duncan, M., Quinn, T., \& Tremaine, S. 1987, AJ, 94, 1330

Duncan, M. J., \& Levison, H. F. 1997, Sci, 276, 1670

Elliot, J. L., Kern, S. D., Clancy, K. B., et al. 2005, AJ, 129, 1117

Eriksson, L. E. J., Mustill, A. J., \& Johansen, A. 2018, MNRAS, 475, 4609

Flaugher, B., Diehl, H. T., Honscheid, K., et al. 2015, AJ, 150, 150

Fornasier, S., Lellouch, E., Müller, T., et al. 2013, A\&A, 555, A15

Fraser, W. C., Brown, M. E., Morbidelli, A., Parker, A., \& Batygin, K. 2014 , ApJ, 782, 100

Fuentes, C. I., \& Holman, M. J. 2008, AJ, 136, 83

Gaia Collaboration, Brown, A. G. A., Vallenari, A., et al. 2016, A\&A, 595, A2

Gallardo, T., Hugo, G., \& Pais, P. 2012, Icar, 220, 392

Gerdes, D. W., Jennings, R. J., Bernstein, G. M., et al. 2016, AJ, 151, 39

Gerdes, D. W., Sako, M., Hamilton, S., et al. 2017, ApJL, 839, L15

Gladman, B., Holman, M., Grav, T., et al. 2002, Icar, 157, 269

Gladman, B., Kavelaars, J., Petit, J.-M., et al. 2009, ApJL, 697, L91

Gladman, B., Marsden, B. G., \& Vanlaerhoven, C. 2008, in The Solar System Beyond Neptune, ed. M. A. Barucci et al. (Tucson, AZ: Univ. Arizona Press), 43

Goldstein, D. A., D'Andrea, C. B., Fischer, J. A., et al. 2015, AJ, 150, 82

Gomes, R., Deienno, R., \& Morbidelli, A. 2017, AJ, 153, 27

Gomes, R., Levison, H. F., Tsiganis, K., \& Morbidelli, A. 2005, Natur, 435,466

Hadden, S., Li, G., Payne, M. J., \& Holman, M. J. 2018, AJ, 155, 249

Holman, M. J., \& Payne, M. J. 2016a, AJ, 152, 80

Holman, M. J., \& Payne, M. J. 2016b, AJ, 152, 94

Holman, M. J., Payne, M. J., Fraser, W., et al. 2018, ApJL, 855, L6

Hunter, J. D. 2007, CSE, 9, 90

Jiménez-Torres, J. J., Pichardo, B., Lake, G., \& Throop, H. 2011, MNRAS, 418,1272

Jones, E., Oliphant, T., Peterson, P., et al. 2001, SciPy: Open Source Scientific Tools for Python, https://www.scipy.org/

Kenyon, S. J., \& Bromley, B. C. 2004, Natur, 432, 598

Kenyon, S. J., \& Bromley, B. C. 2016, ApJ, 825, 33

Kessler, R., Marriner, J., Childress, M., et al. 2015, AJ, 150, 172

Khain, T., Batygin, K., \& Brown, M. E. 2018, AJ, 155, 250 
Kinoshita, H., \& Nakai, H. 1999, CeMDA, 75, 125

Kluyver, T., Ragan-Kelley, B., Pérez, F., et al. 2016, Positioning and Power in Academic Publishing: Players, Agents and Agendas (Clifton, VA: IOS Press)

Kozai, Y. 1962, AJ, 67, 591

Lada, C. J., \& Lada, E. A. 2003, ARA\&A, 41, 57

Lawler, S. M., Kavelaars, J., Alexandersen, M., et al. 2018, FrASS, 5, 14

Lellouch, E., Kiss, C., Santos-Sanz, P., et al. 2010, A\&A, 518, L147

Lellouch, E., Santos-Sanz, P., Lacerda, P., et al. 2013, A\&A, 557, A60

Levison, H. F., Morbidelli, A., Van Laerhoven, C., Gomes, R., \& Tsiganis, K. 2008, Icar, 196, 258

Li, G., \& Adams, F. C. 2015, MNRAS, 448, 344

Li, G., \& Adams, F. C. 2016, ApJL, 823, L3

Lim, T. L., Stansberry, J., Müller, T. G., et al. 2010, A\&A, 518, L148

Lin, H. W., Chen, Y.-T., Holman, M. J., et al. 2016, AJ, 152, 147

Lykawka, P. S., \& Mukai, T. 2006, P\&SS, 54, 87

Madigan, A.-M., \& McCourt, M. 2016, MNRAS, 457, L89

Madigan, A.-M., Zderic, A., McCourt, M., \& Fleisig, J. 2018, arXiv:1805.03651

Malhotra, R., Volk, K., \& Wang, X. 2016, ApJL, 824, L22

Marsden, B. G., Sekanina, Z., \& Everhart, E. 1978, AJ, 83, 64

McKinney, W. 2010, in Proc. 9th Python in Science Conf., ed. S. van der Walt \& J. Millman, 51

Millholland, S., \& Laughlin, G. 2017, AJ, 153, 91

Millis, R. L., Buie, M. W., Wasserman, L. H., et al. 2002, AJ, 123, 2083

Morbidelli, A., Levison, H. F., Tsiganis, K., \& Gomes, R. 2005, Natur, 435,462

Müller, T. G., Lellouch, E., Stansberry, J., et al. 2010, A\&A, 518, L146

Murray, C. D., \& Dermott, S. F. 1999, Solar System Dynamics (Cambridge: Cambridge Univ. Press)

Mustill, A. J., Raymond, S. N., \& Davies, M. B. 2016, MNRAS, 460, L109
Oliphant, T. E. 2006, Guide to NumPy, http://web.mit.edu/dvp/Public/ numpybook.pdf

Parker, R. J., Lichtenberg, T., \& Quanz, S. P. 2017, MNRAS, 472, L75

Pérez, F., \& Granger, B. E. 2007, CSE, 9, 21

Pike, R. E., Fraser, W. C., Schwamb, M. E., et al. 2017, AJ, 154, 101

Pireaux, S., \& Rozelot, J.-P. 2003, Ap\&SS, 284, 1159

Pordes, R., , OSG Consortium, OSG Consortium, Petravick, D., et al. 2007, JPhCS, 78, 012057

Porras, A., Christopher, M., Allen, L., et al. 2003, AJ, 126, 1916

Robutel, P., \& Laskar, J. 2001, Icar, 152, 4

Saillenfest, M., Fouchard, M., Tommei, G., \& Valsecchi, G. B. 2016, CeMDA, 126,369

Saillenfest, M., Fouchard, M., Tommei, G., \& Valsecchi, G. B. 2017, CeMDA, 129,329

Sfiligoi, I. 2008, JPhCS, 119, 062044

Sfiligoi, I., Bradley, D. C., Holzman, B., et al. 2009, WRI World Congress on Computer Science and Information Engineering, Vol. 2, 428

Shankman, C., Kavelaars, J. J., Bannister, M. T., et al. 2017a, AJ, 154, 50

Shankman, C., Kavelaars, J. J., Lawler, S. M., Gladman, B. J., \& Bannister, M. T. 2017b, AJ, 153, 63

Sheppard, S. S., \& Trujillo, C. 2016, AJ, 152, 221

Silsbee, K., \& Tremaine, S. 2018, AJ, 155, 75

Solontoi, M., Ivezić, Ž., Jurić, M., et al. 2012, Icar, 218, 571

Tegler, S. C., \& Romanishin, W. 2000, Natur, 407, 979

Thomas, F., \& Morbidelli, A. 1996, CeMDA, 64, 209

Towns, J., Cockerill, T., Dahan, M., et al. 2014, CSE, 16, 62

Trujillo, C. A., \& Sheppard, S. S. 2014, Natur, 507, 471

Tsiganis, K., Gomes, R., Morbidelli, A., \& Levison, H. F. 2005, Natur, 435,459

Volk, K., Murray-Clay, R. A., Gladman, B. J., et al. 2018, AJ, 155, 260 